\title{
ON THE MODELLING AND MANAGEMENT OF TRAFFIC
}

\author{
Rinaldo M. Colombo ${ }^{1}$, Paola Goatin ${ }^{2}$ and Massimiliano D. Rosini ${ }^{3}$
}

\begin{abstract}
Several realistic situations in vehicular traffic that give rise to queues can be modeled through conservation laws with boundary and unilateral constraints on the flux. This paper provides a rigorous analytical framework for these descriptions, comprising stability with respect to the initial data, to the boundary inflow and to the constraint. We present a framework to rigorously state optimal management problems and prove the existence of the corresponding optimal controls. Specific cases are dealt with in detail through ad hoc numerical integrations. These are here obtained implementing the wave front tracking algorithm, which appears to be very precise in computing, for instance, the exit times.
\end{abstract}

Mathematics Subject Classification. 35L65, 90B20.

Received July 20, 2010. Revised November 24, 2010.

Published online February 23, 2011.

\section{INTRODUCTION}

The evolution of traffic flowing along a highway can be described by the classical Lighthill-Whitham [40] and Richards [46] (LWR) equation

$$
\partial_{t} \rho+\partial_{x} f(\rho)=0 \quad \text { with } \quad f(\rho)=\rho v(\rho),
$$

where $\rho=\rho(t, x)$ denotes the density of vehicles on the highway, the speed $v$ is given through a suitable speed law $v=v(\rho)$ and $f$ is the flow. Equation (1.1) needs to be supplemented with an initial datum $\rho_{o}=\rho_{o}(x)$ and, depending on the situation at hand, also by the time dependent inflow $q_{o}$ at a given entry, say sited at $x=0$, namely $f(\rho(t, 0))=q_{o}(t)$.

Traffic flow is subject to various restrictions, such as toll gates, traffic lights, construction sites, or the effects of accidents. All these situations amount to limit the flow at specific locations along the road and, hence, can be described adding suitable unilateral constraints on the flow, such as $f\left(\rho\left(t, x_{c}\right)\right) \leq q_{c}(t), x_{c}>0$ being the location of the toll gate (or traffic light, or accident...) and $q_{c}=q_{c}(t)$ being the possibly time dependent maximal flow through $x_{c}$ allowed by the toll gate (or traffic light, or accident...).

\footnotetext{
Keywords and phrases. Optimal control of conservation laws, constrained hyperbolic PDEs, traffic modelling.

1 Dipartimento di Matematica, Università degli studi di Brescia, Italia. rinaldo@ing.unibs.it

2 INRIA Sophia Antipolis - Méditerranée, EPI OPALE, France. paola.goatin@inria.fr

3 ICM, Uniwersytet Warszawski, Polska. mrosini@icm.edu.pl
} 
Below, we provide a basic well posedness result for the constrained initial-boundary value problem

$$
\left\{\begin{array}{lc}
\partial_{t} \rho+\partial_{x} f(\rho)=0 & (t, x) \in \mathbb{R}^{+} \times \mathbb{R}^{+} \\
\rho(0, x)=\rho_{o}(x) & x \in \mathbb{R}^{+} \\
f(\rho(t, 0))=q_{o}(t) & t \in \mathbb{R}^{+} \\
f\left(\rho\left(t, x_{c}\right)\right) \leq q_{c}(t) & t \in \mathbb{R}^{+}
\end{array}\right.
$$

extending the results in $[4,11]$, see also [13]. The $\mathbf{L}^{1}$-Lipschitz dependence of the solution to (1.2) with respect to the initial datum $\rho_{o}$, the inflow $q_{o}$ and the constraint $q_{c}$ is proved.

This well posedness result allows to prove the existence of optimal management strategies. Indeed, we propose some criteria to be optimized in a rational control of traffic. Various reasonable goals to be pursued in the management of vehicular traffic can be described through suitable integral functionals, which have to be maximized or minimized along solutions to (1.2). As possible control parameters we consider below the inflow $q_{o}$ or the flow $q_{c}$ at the constraint.

We stress that, in our intentions, the standardized situation (1.2) and the corresponding well posedness results serve as a model situation. In other words, problem (1.2), Theorem 2.2 and Corollary 2.3 constitute the basic bricks for a framework where more complex models can be constructed, used and managed. Indeed, formally different and more complicate situations can be described through a sort of juxtaposition of several simpler problems of the type (1.2). The present analytical results can be then easily applied to each of the simpler problems, giving then information about the full situation. We provide an example of this construction in Section 2.4.

Most of the examples presented below lead to constrained initial-boundary value problems that are numerically integrated. To this aim, we use the wave front tracking method as a numerical algorithm. This technique was first introduced in [16], see also [8,26]. It has proved to be an effective and efficient tool in the analytical study of systems of conservation laws, see for instance [1,2,9,11]. Our choice of using it as a numerical tool is motivated by our need to accurately compute the position of a shock in the solution to a conservation law. As shown in Section 3.1, at least in the particular case considered therein, wave front tracking proves to be precise and efficient.

Our choice of the LWR model is motivated by our aim of providing detailed analytical proofs. We stress that the numerical method used here, i.e. wave front tracking, as well as the general framework described below, may be easily extended substituting the LWR equation with the 2-phase models $[7,10,14,15,19]$ or also with the classical Aw-Rascle model [5,42]. It is natural to conjecture that the results below can be extended to several of these more refined models, however the necessary analytical techniques get more involved. Moreover, the LWR model is still considered in transportation journals, see $[25,27,35,36,39,41]$. In connection with it, we provide an analytical framework and ad hoc numerical techniques to study initial boundary value problems with junctions and unilateral constraints that, to our knowledge, have not been considered before.

In Section 2, we provide a rigorous analytical framework for (1.2) and for related control problems. Various examples are presented in Section 3, while the technical details are deferred to the final Section 4.

\section{AnAlytical FRAMEWORK}

\subsection{The constrained IBVP (1.2)}

Throughout, $R$ denotes the maximal possible traffic density and $\bar{f}$ the maximal traffic flow. $\bar{\rho}$ stands for the density at which the flow $\bar{f}$ is attained. The initial density distribution $\rho_{o}$, the inflow $q_{o}$ and the constraint $q_{c}$ on the flow merely need to be bounded. More precisely, we assume the following condition on the flow in (1.2):

$(\mathbf{F}): f \in \mathbf{C}^{\mathbf{0}, \mathbf{1}}([0, R] ;[0, \bar{f}])$ satisfies $f(0)=f(R)=0$ and there exists a $\left.\bar{\rho} \in\right] 0, R\left[\right.$ such that $f^{\prime}(\rho)(\bar{\rho}-\rho)>0$ for a.e. $\rho \in[0, R]$.

Recall that $\mathbf{C}^{\mathbf{0}, \mathbf{1}}([0, R] ;[0, \bar{f}])$ is the set of Lipschitz continuous functions defined on $[0, R]$ and attaining values in $[0, \bar{f}]$. By $(\mathbf{F}), \bar{\rho}$ is the unique point of maximum of $f$ and $\bar{f}=f(\bar{\rho})=\max _{\rho \in[0, R]} f(\rho)$, see Figure 1 . Moreover, 


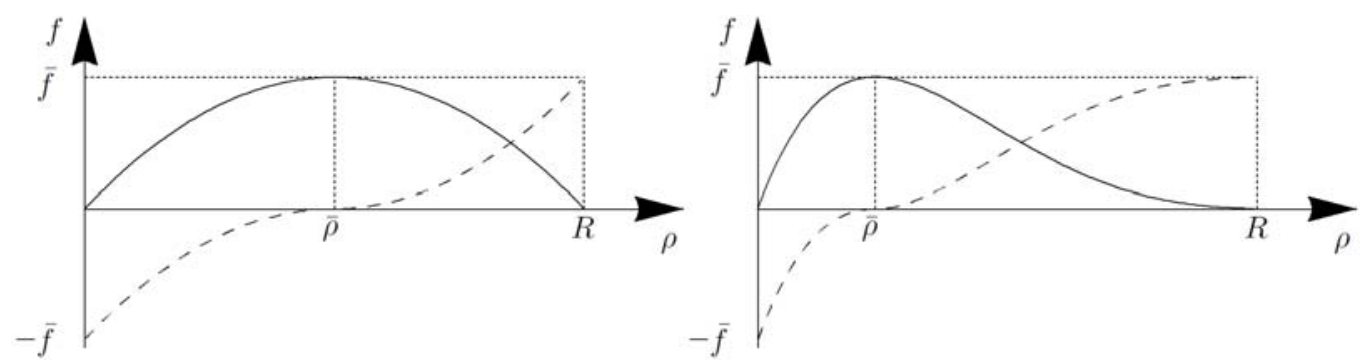

FiguRE 1. Fundamental diagrams satisfying $(\mathbf{F})$. In both diagrams, the solid line represents a flux $f$ satisfying condition $(\mathbf{F})$ and the dashed line represents the corresponding function $\Psi$ given by $(2.2)$.

the restriction $f_{*}$ of $f$ to $[0, \bar{\rho}]$, i.e. $f_{*}=f_{\mid[0, \bar{\rho}]}$, is invertible and its inverse $f_{*}^{-1}$ satisfies

$$
f_{*}^{-1}:[0, \bar{f}] \rightarrow[0, \bar{\rho}] \quad \text { where } \quad f_{*}^{-1}(q)=\rho \Longleftrightarrow \rho \in[0, \bar{\rho}] \text { and } f(\rho)=q \text {. }
$$

The choice of a specific fundamental diagram $f=f(\rho)$ usually relies on the choice of the speed law $v=v(\rho)$, since $f(\rho)=\rho v(\rho)$. This issue has been widely discussed in the literature and the requirement (F) comprises, for instance, all the choices described in [9], Formula (2), [17], Figure 8(a), [21], Formula (13), [22,28,31] and [43], Figure 2 .

A rigorous definition of solution to (1.2) is obtained by suitably merging the definition of solution to an initial-boundary value problem with that of a solution to a scalar conservation law with a unilateral constraint. Usually, in the more analytical literature, the boundary condition amounts to assign the value of the unknown along the boundary, see [1,3,6,47] and [2], Case (C). On the contrary, in (1.2) we assign the inflow, which is better suited to traffic problems. This accounts for the appearance of $f_{*}^{-1}$ in the definition below.

Definition 2.1. A map $\rho \in \mathbf{C}^{\mathbf{0}}\left(\mathbb{R}^{+} ; \mathbf{L}^{\infty}\left(\mathbb{R}^{+} ;[0, R]\right)\right)$ is a weak entropy solution to (1.2) if the following conditions hold:

1. For every test function $\varphi \in \mathbf{C}_{\mathbf{c}}^{\mathbf{1}}\left(\mathbb{R}^{2} ; \mathbb{R}^{+}\right)$and for every $k \in[0, R]$

$$
\begin{aligned}
& \int_{0}^{+\infty} \int_{0}^{+\infty}\left(|\rho-k| \partial_{t} \varphi+\operatorname{sgn}(\rho-k)(f(\rho)-f(k)) \partial_{x} \varphi\right) \mathrm{d} x \mathrm{~d} t \\
& +\int_{0}^{+\infty}\left|\rho_{o}-k\right| \varphi(0, x) \mathrm{d} x \\
& +\int_{0}^{+\infty} \operatorname{sgn}\left(f_{*}^{-1}\left(q_{o}(t)\right)-k\right)(f(\rho(t, 0+))-f(k)) \varphi(t, 0) \mathrm{d} t \\
& +2 \int_{0}^{+\infty}\left(1-\frac{q_{c}(t)}{f(\bar{\rho})}\right) f(k) \varphi\left(t, x_{c}\right) \mathrm{d} t \geq 0 .
\end{aligned}
$$

2. For a.e. $t \in \mathbb{R}^{+}, f\left(\rho\left(t, x_{c}-\right)\right)=f\left(\rho\left(t, x_{c}+\right)\right) \leq q_{c}(t)$.

Above we denote by $\rho\left(t, x_{c} \pm\right)$ the measure theoretic traces implicitly defined by

$$
\begin{aligned}
& \lim _{\varepsilon \rightarrow 0+} \frac{1}{\varepsilon} \int_{0}^{+\infty} \int_{x_{c}}^{x_{c}+\varepsilon}\left|\rho(t, x)-\rho\left(t, x_{c}+\right)\right| \varphi(t, x) \mathrm{d} x \mathrm{~d} t=0 \\
& \lim _{\varepsilon \rightarrow 0+} \frac{1}{\varepsilon} \int_{0}^{+\infty} \int_{x_{c}-\varepsilon}^{x_{c}}\left|\rho(t, x)-\rho\left(t, x_{c}-\right)\right| \varphi(t, x) \mathrm{d} x \mathrm{~d} t=0
\end{aligned}
$$

for all $\varphi \in \mathbf{C}_{\mathbf{c}}^{\mathbf{1}}\left(\mathbb{R}^{2} ; \mathbb{R}\right)$. Remark that both traces at $x_{c}$ in $\mathbf{2}$. exist and are finite, by [4], Theorem 2.2. 
The first two lines in 1. originate from the classical Kružkov definition [34], Definition 1, in the case of the Cauchy problem, i.e. with no boundary and no constraints. The third line is motivated by the boundary, following [6], Formula (17), or [47], Formula (15.14). The latter line accounts for the constraint, as in [11], Definitions 3.1 and 3.2, see also [4], Definition 2.1. For other equivalent formulations in the case of unilateral constraints, we refer to [4], Proposition 2.6.

It is immediate to prove that any solution in the usual sense of [6], Formula (17), to

$$
\left\{\begin{array}{lc}
\partial_{t} \rho+\partial_{x} f(\rho)=0 & (t, x) \in \mathbb{R}^{+} \times \mathbb{R}^{+} \\
\rho(0, x)=\rho_{o}(x) & x \in \mathbb{R}^{+} \\
\rho(t, 0)=f_{*}^{-1}\left(q_{o}(t)\right) & t \in \mathbb{R}^{+}
\end{array}\right.
$$

that satisfies $f\left(\rho\left(t, x_{c}-\right)\right)=f\left(\rho\left(t, x_{c}+\right)\right) \leq q_{c}(t)$, also satisfies Definition 2.1. Similarly, by the local nature of Definition 2.1, any solution to (1.2) in the sense of Definition 2.1 also solves (2.1) in the sense of [6], Formula (17), in a neighborhood of any $(t, x) \in \mathbb{R}^{+} \times \mathbb{R}^{+}$, with $x \neq x_{c}$. Refer to [11] for a discussion of the nonclassical, or non entropic, shocks that may arise at $x_{c}$ due to the presence of the constraint.

As in [11], Formula (3.1) and [49], we introduce the nonlinear map

$$
\Psi(\rho)=\operatorname{sgn}(\rho-\bar{\rho})(\bar{f}-f(\rho))
$$

which plays a key role throughout the analytical construction below, see Figure 1. First, it allows to introduce the domain

$$
\mathcal{D}=\left\{\rho \in \mathbf{L}^{\mathbf{1}}\left(\mathbb{R}^{+} ;[0, R]\right): \Psi(\rho) \in \mathbf{B V}\left(\mathbb{R}^{+} ; \mathbb{R}\right)\right\}
$$

and, second, it allows to state and prove the following well posedness theorem.

Theorem 2.2. Let (F) hold. Assume moreover that:

(R0): $\rho_{o} \in \mathcal{D}$,

(Q): $q_{o}, q_{c} \in \mathbf{B V}\left(\mathbb{R}^{+} ;[0, \bar{f}]\right)$.

Then, there exists a unique solution $\rho=\rho\left(t, x ; \rho_{o}, q_{o}, q_{c}\right)$ to $(1.2)$ in the sense of Definition 2.1 and, for all $t \in \mathbb{R}^{+}, \rho\left(t ; \rho_{o}, q_{o}, q_{c}\right) \in \mathcal{D}$. Moreover, if $\rho_{o}, \rho_{o}^{\prime}$ both satisfy (R0), $q_{o}, q_{o}^{\prime}$ and $q_{c}, q_{c}^{\prime}$ satisfy (Q), the following Lipschitz estimate on the corresponding solutions $\rho, \rho^{\prime}$ holds, for every $t>0$ :

$$
\left\|\rho(t)-\rho^{\prime}(t)\right\|_{\mathbf{L}^{1}\left(\mathbb{R}^{+} ; \mathbb{R}\right)} \leq\left\|\rho_{o}-\rho_{o}^{\prime}\right\|_{\mathbf{L}^{1}\left(\mathbb{R}^{+} ; \mathbb{R}\right)}+\left\|q_{o}-q_{o}^{\prime}\right\|_{\mathbf{L}^{1}([0, t] ; \mathbb{R})}+2\left\|q_{c}-q_{c}^{\prime}\right\|_{\mathbf{L}^{1}([0, t] ; \mathbb{R})} .
$$

The proof is deferred to Section 4.

Further stability estimates are necessary, in view of the optimal control problem considered in Section 3.2. The following lemma ensures that the through flow at a point $\bar{x}$ is a Lipschitz function in $\mathbf{L}^{\mathbf{1}}$ of the inflow $q_{o}$ and of the constraint $q_{c}$.

Corollary 2.3. Let $f$ satisfy $(\mathbf{F}), \rho_{o} \equiv 0$ and let $q_{o}, q_{o}^{\prime}, q_{c}, q_{c}^{\prime}$ all satisfy $(\mathbf{Q})$. Assume that there exists $\tau_{o}$ such that $\operatorname{spt}\left(q_{o}\right) \cup \operatorname{spt}\left(q_{o}^{\prime}\right) \subseteq\left[0, \tau_{o}\right]$ and that there exists a positive $\tau_{c}$ such that

$$
\int_{0}^{\tau_{c}} f\left(\rho\left(t, x_{c}+\right)\right) \mathrm{d} t=\int_{0}^{\tau_{o}} q_{o}(t) \mathrm{d} t \quad \text { and } \quad \int_{0}^{\tau_{c}} f\left(\rho^{\prime}\left(t, x_{c}+\right)\right) \mathrm{d} t=\int_{0}^{\tau_{o}} q_{o}^{\prime}(t) \mathrm{d} t
$$

where $\rho$ and $\rho^{\prime}$ are the weak entropy solutions to (1.2) corresponding, respectively, to $q_{o}, q_{c}$ and to $q_{o}^{\prime}, q_{c}^{\prime}$. Then, for all $x>x_{c}$ and $t \geq 0$, the following Lipschitz estimate holds:

$$
\begin{aligned}
\left\|f(\rho(\cdot, x-))-f\left(\rho^{\prime}(\cdot, x-)\right)\right\|_{\mathbf{L}^{1}([0, t] ; \mathbb{R}) \leq} \leq & \left\|q_{o}-q_{o}^{\prime}\right\|_{\mathbf{L}^{1}\left(\left[0, \min \left\{t, \tau_{o}\right\}\right] ; \mathbb{R}\right)} \\
& +2\left\|q_{c}-q_{c}^{\prime}\right\|_{\mathbf{L}^{1}\left(\left[0, \min \left\{t, \tau_{c}\right\}\right] ; \mathbb{R}\right)} .
\end{aligned}
$$




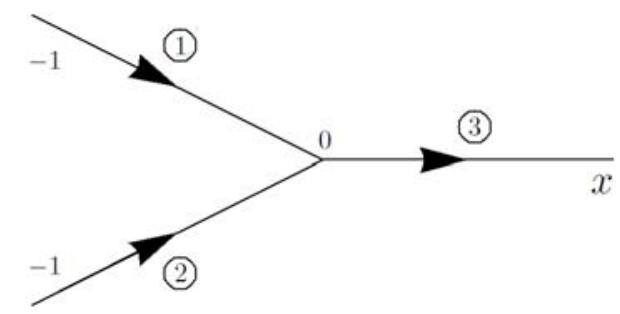

Figure 2. Two roads merging into a third one, as considered in (2.7).

The proof is deferred to Section 4. Above, assumption (2.5) means that all the vehicles entered through 0 are allowed to pass through $x_{c}$ within time $\tau_{c}$.

\subsection{Roads merging}

As an example of how the theory of the previous section can be used in different situations, consider the case in which two (one-way) roads, say (1) and (2), enter through a junction into road (3), see Figure 2. For a thorough treatment of junctions through the LWR model, refer to [45]. Traffic along road (i) is described by an LWR model of the type (1.1) with flow $f=f_{i}(\rho)$, for $i=1,2,3$. The junction, say at $x=0$, is regulated so that the flow from road (i) into (3) is bounded by $q_{i}(t)$, for $i=1,2$. We are thus lead to consider the problem

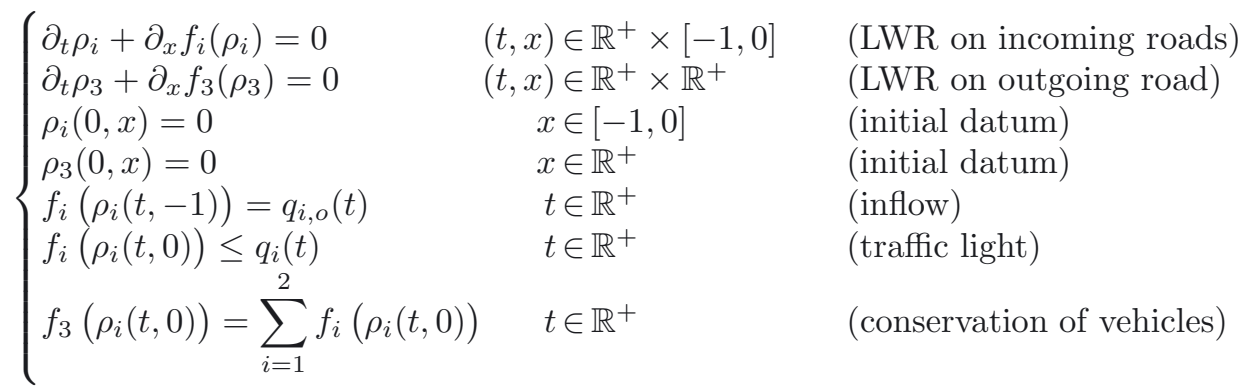

where $i=1,2$. Using the same notation as in the preceding section, we require that the regulation at the junction is reasonable, i.e.

$$
q_{1}(t)+q_{2}(t) \leq \bar{f}_{3} \quad \text { for all } t \geq 0
$$

where $\bar{f}_{3}$ is the maximum of the flow along road (3), consistently with (F). In other words, the total outflow from roads (1) and (2) is regulated so that it never exceeds the maximal possible inflow into road (3). Note that, clearly, this condition does not avoid the formation of queues at the end of roads (1) and (2), before $x=0$.

We remark that, in the framework of (2.7), condition (2.8) provides all the necessary information to obtain a complete model, as shown in Corollary 2.4. Constraint (2.8) thus singles out a unique way to allocate the downstream supply to the upcoming roads. This choice is alternative to various other constructions, see for instance [45] and the references therein in the applied mathematics literature, or [27,29] (which are referred to the LWR model) and [24] in the transport engineering literature. For a related approach to a merging problem with a special consideration of the capacity drop phenomenon, we refer to [23].

Assuming (2.8) and with the regularity assumptions (F) and (Q) on each road, stating and proving the well posedness of (2.7) amount to a repeated application of the arguments in Theorem 2.2.

Corollary 2.4. Let $f_{1}, f_{2}, f_{3}$ satisfy $(\mathbf{F})$ and $q_{i, o}, q_{i}$ satisfy $(\mathbf{Q})$ for $i=1,2$. Assume that (2.8) holds. Then, there exists a unique solution

$$
\begin{array}{rlrl}
\rho_{i} & =\rho_{i}\left(t, x ; q_{i, o}, q_{i}\right) & (t, x) \in \mathbb{R}^{+} \times[-1,0] & i=1,2 \\
\rho_{3}=\rho_{3}\left(t, x ; q_{1, o}, q_{2, o}, q_{1}+q_{2}\right) & (t, x) \in \mathbb{R}^{+} \times \mathbb{R}^{+} &
\end{array}
$$


to (2.7). Finally, if $q_{i, o}^{\prime}, q_{i}^{\prime}$ also satisfy (Q) for $i=1,2$, then the following Lipschitz estimates on the corresponding solutions $\rho, \rho^{\prime}$ hold, for every $t>0$ :

$$
\begin{aligned}
\left\|\rho_{i}(t)-\rho_{i}^{\prime}(t)\right\|_{\mathbf{L}^{1}([-1,0] ; \mathbb{R})} & \leq\left\|q_{i, o}-q_{i, o}^{\prime}\right\|_{\mathbf{L}^{\mathbf{1}}([0, t] ; \mathbb{R})}+2\left\|q_{i}-q_{i}^{\prime}\right\|_{\mathbf{L}^{1}([0, t] ; \mathbb{R})} \quad i=1,2 \\
\left\|\rho_{3}(t)-\rho_{3}^{\prime}(t)\right\|_{\mathbf{L}^{1}(\mathbb{R}+; \mathbb{R})} & \leq \sum_{i=1}^{2}\left(\left\|q_{i, o}-q_{i, o}^{\prime}\right\|_{\mathbf{L}^{1}([0, t] ; \mathbb{R})}+2\left\|q_{i}-q_{i}^{\prime}\right\|_{\mathbf{L}^{1}([0, t] ; \mathbb{R})}\right) .
\end{aligned}
$$

Above, by solution we mean the obvious adaptation of Definition 2.1 to the case (2.7). The proof is deferred to Section 4 and consists of recursive applications of Theorem 2.2 .

Obviously, the insertion of junctions or other constraints to the right of $x=0$ simply amounts to further applications of Theorem 2.2 .

\subsection{Cost functionals}

We now consider some reasonable cost functionals. We prove that their regularity, when computed along the solutions to problems of the type (1.2) or (2.7), is sufficient to ensure the existence of an optimal management strategy. All proofs are deferred to Section 4.

Queue length. First, we rigorously define the functional assigning to a solution to (1.2) the length of the queue before the obstacle at $x_{c}$. Then, we prove its lower semicontinuity and show through an example that upper semicontinuity does not hold. Therefore, this functional can be maximized but hardly minimized. For an engineering approach to the same problem, see for instance [41].

We consider the case in which the through flow at $x_{c}$ is constant, i.e. $q_{c}(t) \equiv Q_{c}$ for a fixed $Q_{c} \in[0, \bar{f}]$. To this aim, we introduce the set

$$
A_{c}(\rho)=\left\{x \in \left[0, x_{c}\left[: \Psi ( \rho ( \xi + ) ) = \overline { f } - Q _ { c } \text { for a.e. } \xi \in \left[x, x_{c}[\}\right.\right.\right.\right.
$$

Note that $A_{c}(\rho)$ is well defined as soon as $\rho \in \mathcal{D}$, with $\mathcal{D}$ as in $(2.3)$. The segment $A_{c}(\rho)$ is the set of points immediately before $x_{c}$, where the traffic flows at the maximal possible level allowed by the constraint $Q_{c}$.

Define now the functional $\mathcal{L}: \mathcal{D} \rightarrow \mathbb{R}^{+}$assigning to any traffic density $\rho \in \mathcal{D}$ the length of the queue before $x_{c}$ by

$$
\mathcal{L}(\rho)= \begin{cases}x_{c}-\inf A_{c}(\rho) & \text { if } A_{c}(\rho) \neq \emptyset \\ 0 & \text { if } A_{c}(\rho)=\emptyset\end{cases}
$$

In the general case where $\rho=\rho(t)$ varies with time, also the queue length is time dependent: $\mathcal{L}=\mathcal{L}(\rho(t))$. Refer to Figure 3 for two visualizations of $\mathcal{L}(\rho)$ in the $(t, x)$-plane, on a solution to (1.2).

Remark that $\mathcal{L}(\rho)$ measures the length of the queue due to the presence of the constraint at $x_{c}$. If further obstacles hinder the flow of traffic lowering the traffic flow below $Q_{c}$, then $\mathcal{L}$ may even vanish.

Proposition 2.5. The map $\mathcal{L}$ at (2.10) is upper semicontinuous with respect to the $\mathbf{L}^{\mathbf{1}}$-norm.

The proof is deferred to Section 4.

In general, the above regularity is not sufficient to ensure the existence of minimizers for the queue length, as the following example shows. Consider problem (1.2). Fix $\left.Q_{c} \in\right] 0, \bar{f}[$ and define the constant inflows $q_{o}^{n}=Q_{c}-\frac{1}{n}, q_{o}^{\infty}=Q_{c}$ and the initial data $\rho_{o}^{n}=f_{*}^{-1}\left(q_{o}^{n}\right), \rho_{o}^{\infty}=f_{*}^{-1}\left(Q_{c}\right)$. Call $\rho^{n}, \rho^{\infty}$ the corresponding solutions to (1.2). Then, for any $n$, by $(2.10)$ we have $\mathcal{L}\left(\rho^{n}(t)\right)=0$. However, for all $t>0$, we have $\mathcal{L}\left(\rho^{\infty}(t)\right)=x_{c}>0$, coherently with Proposition 2.5 and showing that lower continuity may fail.

Stop \& go waves. A relevant criterion in the management of traffic dynamics is the minimization of stop $\mathcal{G}^{\circ}$ go waves, see for instance [30], [33], Chapter 8, and the references therein, [50]. A rigorous definition of these phenomenon, with the similar ones called synchronized traffic, localized clusters or oscillating congested traffic, 


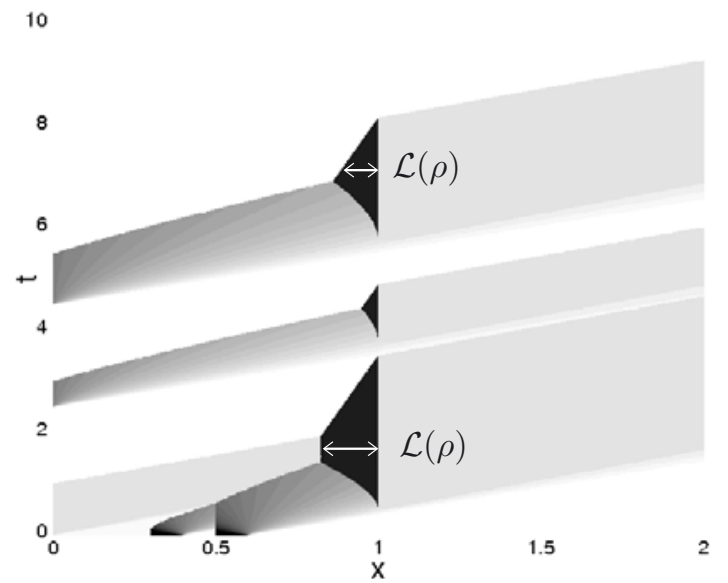

Figure 3. A typical solution to (1.2), portrayed on the $(t, x)$-plane, with two square waves in the initial datum, three waves as inflow from $x=0$ and a constraint at $x_{c}=1$. The length $\mathcal{L}(\rho)$ at two different times is shown.

see $[32,37,51]$ and the overview in [48], is usually substituted by suitable phenomenological descriptions. From the analytical point of view, it is obvious that these waves need to be associated with a high total variation of the speed (for the definition of total variation, see for instance [8], Sect. 2.5).

Hence, reducing stop \& go waves analytically amounts to minimize the total variation of traffic speed. This leads to consider the functional

$$
J(\rho)=\int_{0}^{T} \int_{\mathbb{R}^{+}} p(x) \mathrm{d}\left|\partial_{x} v(\rho)\right| \mathrm{d} t
$$

measuring on the time interval $[0, T]$ the total variation $\left|\partial_{x} v(\rho)\right|$ of the traffic speed $v(\rho)$ weighted by a weight $p(x) \in[0,1]$, higher in more dangerous road segments. Recall that $v$ is a Lipschitz function, by hypothesis $(\mathbf{F})$. Hence, for any $t$, as soon as $\rho(t) \in \mathbf{B V}$, also the function $x \rightarrow v(\rho(t, x))$ is in $\mathbf{B V}$ and its derivative $\partial_{x} v(\rho)$ is a Radon measure on $\mathbb{R}$. The inner integral in (2.11) is thus the integral of $p$ with respect to the time dependent total variation measure $\left|\partial_{x} v(\rho)\right|$. The lower semicontinuity of the functional $J$ in $(2.11)$ follows from [12], Lemma 2.1.

Travel times. From the point of view of drivers, key quantities determining the quality of traffic are related to the time necessary to reach the destination. Refer to (1.2): neglecting the initial datum, i.e. let $\rho_{o}=0$, assume that the inflow $q_{o}$ is non zero and with support contained in, say, $\left[0, \tau_{o}\right]$. The total quantity of vehicles entering the road is thus $Q_{\text {in }}=\int_{0}^{\tau_{o}} q_{o}(t) \mathrm{d} t$, which is assumed to be finite and, obviously, strictly positive. Then, the mean arrival time at $x=\bar{x}>0$ is

$$
T_{a}(\bar{x})=\frac{1}{Q_{\text {in }}} \int_{0}^{+\infty} t f(\rho(t, \bar{x})) \mathrm{d} t,
$$

see also [3], Formula (5.3), where $\rho=\rho(t, x)$ is the solution to (1.2). The mean travel time between the points 0 and $\bar{x}$ can then be easily computed:

$$
T_{t}(\bar{x})=\frac{1}{Q_{\text {in }}} \int_{0}^{+\infty} t(f(\rho(t, \bar{x}))-f(\rho(t, 0))) \mathrm{d} t .
$$

To evaluate the regularity of the functionals (2.12) and (2.13), we consider below the case in which the flow at $x_{o}=0$ is assigned as boundary data, so that $f(\rho(t, 0))=q_{o}(t)$. The notations are the same as those of Corollary 2.3, in particular $\tau_{c}$ is a positive time satisfying (2.5). 
Proposition 2.6. Under the same assumptions and notations of Corollary 2.3, the mean arrival times $T_{a}(\bar{x})$, $T_{a}^{\prime}(\bar{x})$ and the mean travel times $T_{t}(\bar{x}), T_{t}^{\prime}(\bar{x})$ on the segment $[0, \bar{x}]$ satisfy the following Lipschitz estimates:

$$
\begin{aligned}
\left|T_{a}(\bar{x})-T_{a}^{\prime}(\bar{x})\right| \leq & \frac{\tau_{c}}{\min \left\{Q_{\mathrm{in}}, Q_{\mathrm{in}}^{\prime}\right\}}\left(1+\frac{\tau_{c} \bar{f}}{2 \min \left\{Q_{\mathrm{in}}, Q_{\mathrm{in}}^{\prime}\right\}}\right)\left\|q_{o}-q_{o}^{\prime}\right\|_{\mathbf{L}^{1}\left(\left[0, \tau_{o}\right] ; \mathbb{R}\right)} \\
& +\frac{2 \tau_{c}}{\min \left\{Q_{\mathrm{in}}, Q_{\mathrm{in}}^{\prime}\right\}}\left\|q_{c}-q_{c}^{\prime}\right\|_{\mathbf{L}^{1}\left(\left[0, \tau_{c}\right] ; \mathbb{R}\right)} \\
\left|T_{t}(\bar{x})-T_{t}^{\prime}(\bar{x})\right| \leq & \frac{\tau_{c}}{\min \left\{Q_{\mathrm{in}}, Q_{\mathrm{in}}^{\prime}\right\}}\left(2+\frac{\tau_{c} \bar{f}}{\min \left\{Q_{\mathrm{in}}, Q_{\mathrm{in}}^{\prime}\right\}}\right)\left\|q_{o}-q_{o}^{\prime}\right\|_{\mathbf{L}^{1}\left(\left[0, \tau_{o}\right] ; \mathbb{R}\right)} \\
& +\frac{2 \tau_{c}}{\min \left\{Q_{\mathrm{in}}, Q_{\mathrm{in}}^{\prime}\right\}}\left\|q_{c}-q_{c}^{\prime}\right\|_{\mathbf{L}^{1}\left(\left[0, \tau_{c}\right] ; \mathbb{R}\right)} .
\end{aligned}
$$

The proof is deferred to Section 4. Remark that evaluating the exit time $T_{e}$, i.e. the time at which no more vehicle are left in the segment $\left[0, x_{c}\right]$, yields a possibly discontinuous functional, see Figure 6 , right.

Density dependent functionals. A further class of integral functionals of obvious interest is that of integrals of functions of the density, say

$$
\mathcal{F}(\rho)=\int_{0}^{T} \int_{a}^{b} \varphi(\rho(t, x)) w(t, x) \mathrm{d} x \mathrm{~d} t
$$

for a time $T>0$ and points $b>a>0$. As soon as the weight $w$ and the cost function $\varphi$ are continuous, it is immediate to prove that $\mathcal{F}$ is also continuous. This, together with Theorem 2.2, through an application of Weierstraß Theorem, allows to prove the existence of choices of the initial/boundary data and/or of the constraint that optimize $\mathcal{F}$.

Reasonable examples of choices of the function $\varphi$ are:

- To have all vehicles travel at a speed as near as possible to a desired optimal speed $\bar{v}$ along a given road segment $[a, b]$, choose for instance $\varphi(\rho)=(v(\rho)-\bar{v})^{2}$;

- To maximize the traffic flow along $[a, b]$, choose $\varphi(\rho)=f(\rho)$.

\section{EXAMPLES}

The following numerical simulations were obtained by means of the wave front tracking algorithm, see [8,26], the classical Lax-Friedrichs method, see [38], Section 12.5, being used only for comparisons. The next section justifies this choice.

\subsection{The simplest case}

Assume that along a road, at some point $x_{c}$, an obstacle hinders the flow of traffic. This situation can be described through the equations

$$
\left\{\begin{array}{l}
\partial_{t} \rho+\partial_{x} f(\rho)=0 \\
\rho(0, x)=\rho_{o}(x) \\
f\left(\rho\left(t, x_{c}\right)\right) \leq q_{c}(t),
\end{array}\right.
$$

where $\rho_{o}$ represents the initial density and $q_{c}$ the maximal flow at the obstacle. For simplicity, in Figure 4 we choose the speed law $v(\rho)=1-\rho$, see [22], and we take $x_{c}=0, \rho_{o}=\chi_{[-0.9,-0.3]}, q_{c} \equiv 0.2$. Then, the exact solution can be explicitly computed via an elementary application of the wave front tracking method. In particular, using the conservation of $\rho$, we obtain the following exact expression for the exit time $T_{e}$, 


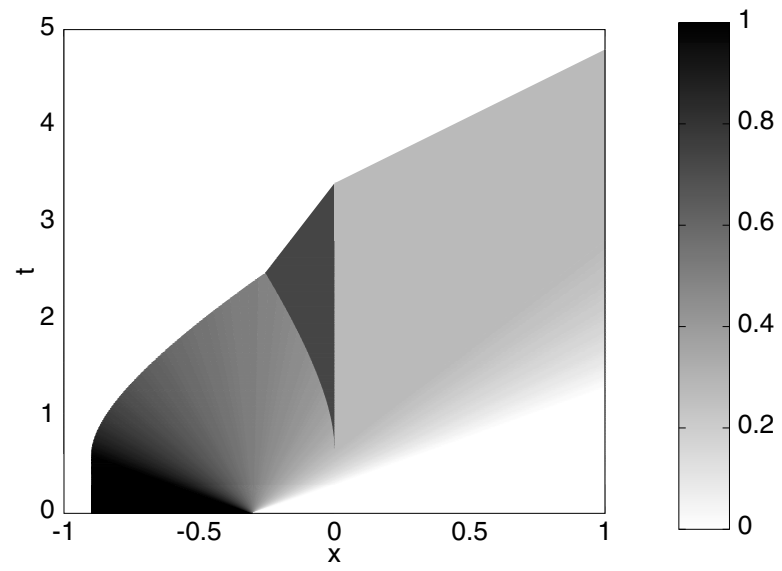

Figure 4. The $(x, t)$-plane showing the numerical integration of $(3.1)$ with $x_{c}=0, \rho_{o}=$ $\chi_{[-0.9,-0.3]}, q_{c}=0.2$. The darker regions represent areas with higher density.

TABLE 1. Results and CPU times of the numerical integration of $(3.1)$ with $v(\rho)=1-\rho$, $x_{c}=0, \rho_{o}=\chi_{[-0.9,-0.3]}, q_{c} \equiv 0.2$ and different numerical parameters, with the wave front tracking algorithm and with the Lax-Friedrichs method (with CFL = 0.5). The solution is portrayed in Figure 4, while the exact exit time is given in (3.2).

\begin{tabular}{|c|c|c|c|c|}
\hline \multirow{8}{*}{$\begin{array}{l}\text { Wave } \\
\text { front } \\
\text { tracking }\end{array}$} & $\Delta \rho$ & Exit time & CPU time $(\mathrm{s})$ & Relative error \\
\hline & 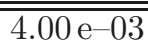 & 4.79564272 & 些0.32 & $=-1.90 \mathrm{e}-02 \%$ \\
\hline & $2.00 \mathrm{e}-03$ & 4.79615273 & 0.59 & $-8.40 \mathrm{e}-03 \%$ \\
\hline & $1.00 \mathrm{e}-03$ & 4.79640870 & 1.18 & $-3.07 \mathrm{e}-03 \%$ \\
\hline & $5.00 \mathrm{e}-04$ & 4.79653693 & 2.36 & $-3.94 \mathrm{e}-04 \%$ \\
\hline & $2.50 \mathrm{e}-04$ & 4.79660132 & 4.95 & $9.49 \mathrm{e}-04 \%$ \\
\hline & $1.25 \mathrm{e}-04$ & 4.79656903 & 10.60 & $2.76 \mathrm{e}-04 \%$ \\
\hline & $6.25 \mathrm{e}-05$ & 4.79655291 & 24.48 & $-6.06 \mathrm{e}-05 \%$ \\
\hline
\end{tabular}

\begin{tabular}{|c|c|c|c|c|}
\hline \multicolumn{1}{|c|}{$\Delta x$} & Exit time & CPU time $(\mathrm{s})$ & Relative error \\
\hline \hline \multirow{4}{*}{ Lax-Friedrichs } & $4.00 \mathrm{e}-03$ & 4.94600000 & 1.69 & $3.12 \mathrm{e}-00 \%$ \\
\cline { 2 - 5 } & $2.00 \mathrm{e}-03$ & 4.87000000 & 5.18 & $1.53 \mathrm{e}-00 \%$ \\
\cline { 2 - 5 } & $1.00 \mathrm{e}-03$ & 4.83300000 & 18.90 & $7.60 \mathrm{e}-01 \%$ \\
\cline { 2 - 5 } & $5.00 \mathrm{e}-04$ & 4.81475000 & 73.40 & $3.79 \mathrm{e}-01 \%$ \\
\cline { 2 - 5 } & $2.50 \mathrm{e}-04$ & 4.80562500 & 295.99 & $1.89 \mathrm{e}-01 \%$ \\
\hline $1.25 \mathrm{e}-04$ & 4.80100000 & 1213.41 & $9.27 \mathrm{e}-02 \%$ \\
\hline $6.25 \mathrm{e}-05$ & 4.79878125 & 5264.29 & $4.64 \mathrm{e}-02 \%$ \\
\hline
\end{tabular}

i.e. the time at which the last vehicle passes through $x=1$ :

$$
T_{e}=\frac{25}{4}-\frac{13}{4 \sqrt{5}} \quad(\approx 4.7965558146 \ldots)
$$



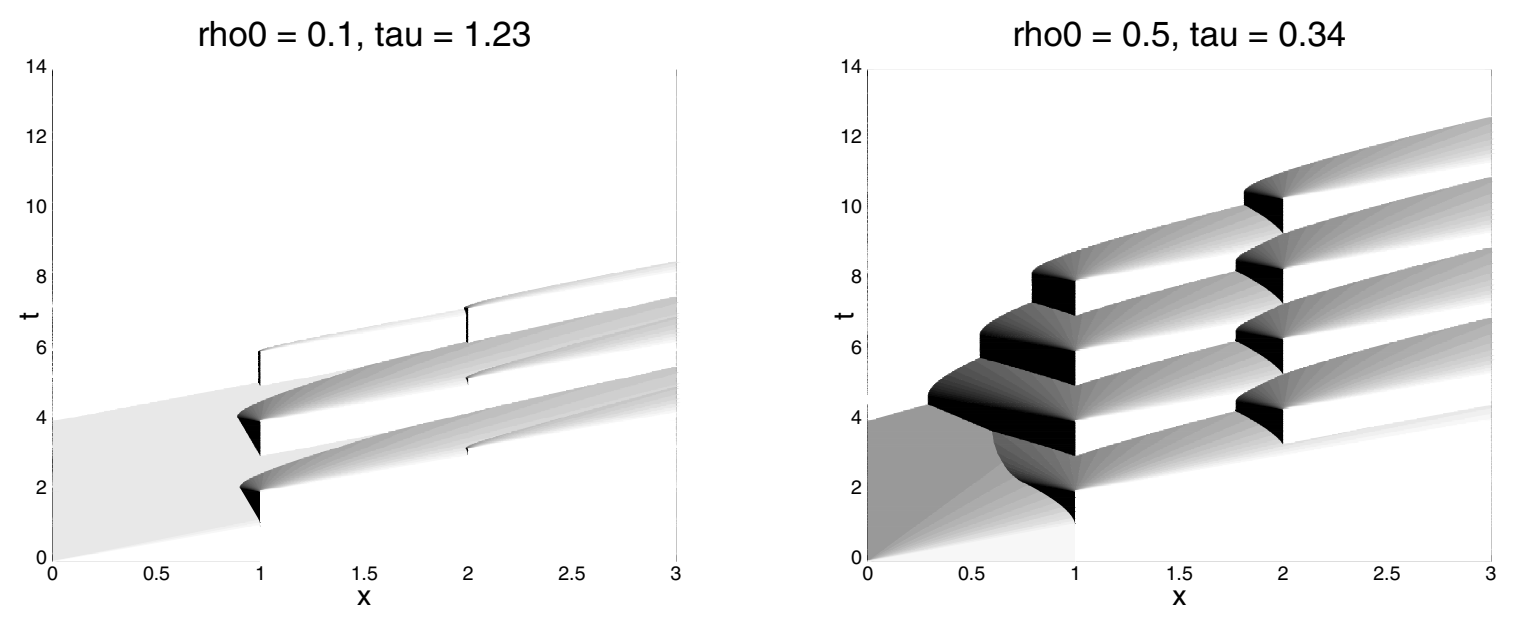

Figure 5. Two solutions to (3.3), corresponding to $\rho_{o}=0.1, \tau=1.23$, left, and $\rho_{o}=0.5$, $\tau=0.34$, right. The corresponding mean arrival times and exit times are part of Figure 6 .

To numerically integrate (3.1) we used both the wave front tracking and the Lax-Friedrichs methods, the latter adapted to the present constrained situation as in [4]. The results are summarized in Table 1. Remark that, in the case of the wave front tracking scheme, we discretize the $\rho$ variable, so that the mesh size has to be understood in $\rho$ units. On the other hand, as it is more usual in the numerics for conservation laws, the LaxFriedrichs scheme relies on the discretization of the space and time variables. It is important to observe that the choice of the initial datum may strongly affect the CPU time in the case of the wave front tracking algorithm. Indeed the number of operations carried out with this method depends on the number of waves, which in turn is determined by the initial datum. On the contrary, the number of operations in the Lax-Friedrichs method only depends on the mesh size.

We stress however that the use of the wave front tracking method allows for a much greater precision, in spite of the fact that the exit time is, in general, not a continuous function, see Figure 6, right. Indeed, most numerical methods introduce viscosity or averaging of the $\rho$ variable. The wave front tracking method, by its nature, computes piecewise constant solutions and keeps track of the location of the points of jump. Thus, in computing quantities such as the exit time, wave front tracking appears particularly suitable.

\subsection{Synchronizing traffic lights}

Consider the segment $[0,3]$ of a road $\mathbb{R}^{+}$with two traffic lights, one at $x_{b}=1$ and one at $x_{c}=2$, respectively characterized by maximal flows $q_{b}$ and $q_{c}$. We use the standard speed law $v(\rho)=1-\rho$. At $x=0$ the inflow $q_{o}$ is constant throughout the time interval $[0,4]$ and then vanishes. In Figure 5, we choose the sample values $q_{o}=f\left(\rho_{o}\right)$, with $\rho_{o}=0.01,0.1,0.2,0.3,0.4,0.5$. Consider the following refinement of $(1.2)$

$$
\left\{\begin{array} { l r l } 
{ \partial _ { t } \rho + \partial _ { x } f ( \rho ) = 0 } & { ( t , x ) \in \mathbb { R } ^ { + } \times \mathbb { R } ^ { + } } \\
{ \rho ( 0 , x ) = 0 } & { x \in \mathbb { R } ^ { + } } \\
{ f ( \rho ( t , 0 ) ) = q _ { o } ( t ) } & { t \in \mathbb { R } ^ { + } } \\
{ f ( \rho ( t , x _ { b } ) ) \leq q _ { b } ( t ) } & { t \in \mathbb { R } ^ { + } } \\
{ f ( \rho ( t , x _ { c } ) ) \leq q _ { c } ( t ) } & { t \in \mathbb { R } ^ { + } }
\end{array} \quad \left\{\begin{array}{l}
q_{o}(t)=f\left(\rho_{o}\right) \chi_{[0,4]}(t) \\
\rho_{o}(t)=0.01,0.1,0.2,0.3,0.4,0.5 \\
q_{b}(t)=0.25 \chi_{[0,1] \cup[2,3] \cup[4,5] \cup[6,7]}(t) \\
q_{c}^{\tau}(t)=q_{b}(t-\tau)
\end{array}\right.\right.
$$

to describe the evolution of traffic. Assume that the two traffic lights have the same fixed frequency of red/green light, say 1 time unit for each regime, so that $q_{c}^{\tau}(t)=q_{b}(t-\tau)$ for a delay $\tau \in\left[0,2\left[\right.\right.$. Moreover, we set $q_{c}^{\tau}(t)=0$ during the red interval and $q_{c}^{\tau}(t)=\bar{f}=0.25$ during the green one. 

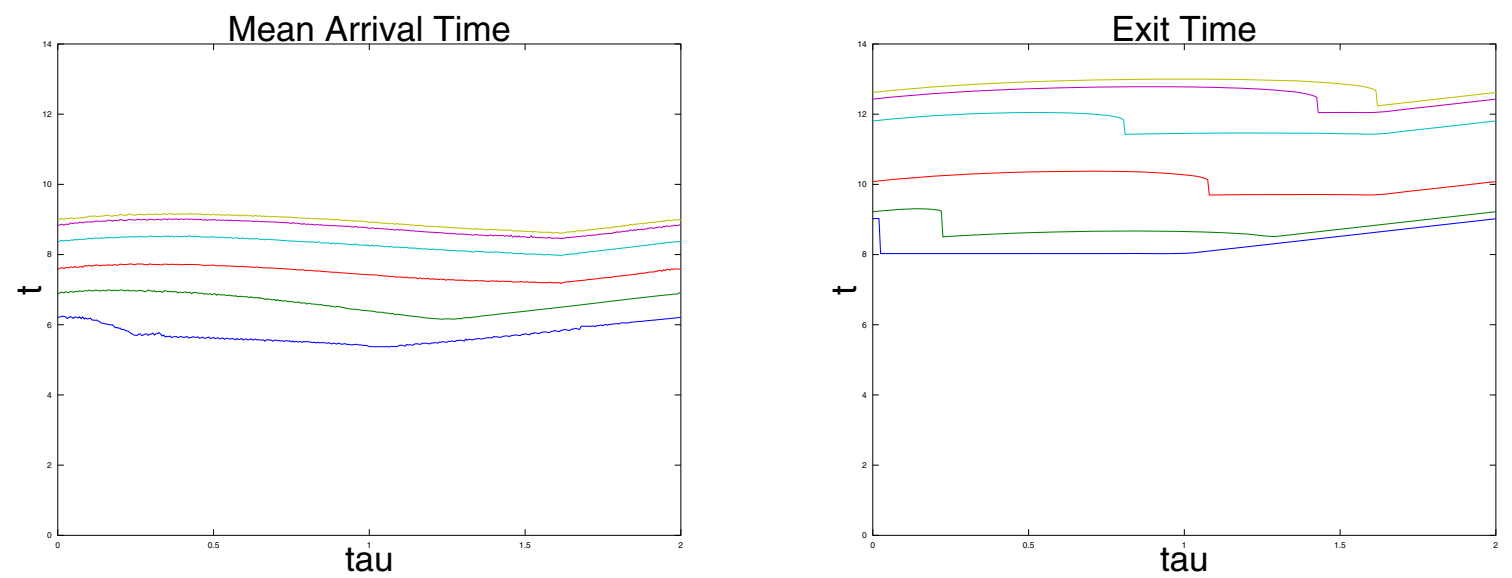

FiguRE 6. Left, mean arrival times and, right, exit times as functions of $\tau$. Both graphs refer to (3.3), with the lower graphs corresponding to the lower inflows. Coherently with Proposition 2.6, the functions on the left are Lipschitz continuous, whereas the ones on the right display jump discontinuities. Two particular solutions are in Figure 5.

We seek the optimal synchronization of the two traffic lights. The existence of such an optimal delay $\tau$ is ensured by the analytical results in Section 2, through Weierstraß Theorem. Indeed, Theorem 2.2 can easily be extended to the case of (3.3). The map $\tau \rightarrow q_{c}^{\tau}$ is continuous in $\mathbf{L}^{\mathbf{1}}$ and the mean travel time $T_{t}$ defined in (2.13) is an $\mathbf{L}^{\mathbf{1}}$-continuous function of the constraint, by Proposition 2.6.

To estimate this optimal delay, we integrate $(3.3)$ with $\tau=0,0.05,0.10,0.15, \ldots, 0.50$. The graphs of the resulting mean arrival time $T_{a}$ and exit time $T_{e}$ is in Figure 6.

\section{TeChnicAl Details}

Preliminary to the following proof, we adapt the definition of solution to the Riemann problem at the boundary to the present situation, see [2], Definition C, and [20], Section 1. Consider the problem

$$
\left\{\begin{array}{lc}
\partial_{t} \rho+\partial_{x} f(\rho)=0 & (t, x) \in \mathbb{R}^{+} \times \mathbb{R}^{+} \\
f(\rho(t, 0))=q^{l} & t \in \mathbb{R}^{+} \\
\rho(0, x)=\rho^{r} & x \in \mathbb{R}^{+} .
\end{array}\right.
$$

By solution to (4.1) we mean the restriction to $\mathbb{R}^{+} \times \mathbb{R}^{+}$of the Lax solution to the Riemann problem

$$
\left\{\begin{array}{l}
\partial_{t} \rho+\partial_{x} f(\rho)=0 \\
\rho(0, x)= \begin{cases}f_{*}^{-1}\left(q^{l}\right) & x<0 \\
\rho^{r} & x>0\end{cases}
\end{array}\right.
$$

Proof of Theorem 2.2. We follow the procedure in [11], Section 4.2, to deal with the constraints $q_{o}, q_{c}$, while the characteristic boundary is tackled as in $[1,2,18,20]$, for instance.

Fix a strictly positive $n \in \mathbb{N}$ and introduce in $[0, R]$ the mesh $\mathcal{M}_{n}$ defined by $\mathcal{M}_{n}=f^{-1}\left(2^{-n} \mathbb{N}\right) \cup\{\bar{\rho}\}$. Let PLC $([0, R] ;[0, \bar{f}])$ be the set of piecewise linear and continuous functions defined on $[0, R]$, whose derivative exists in $] 0, R\left[\backslash \mathcal{M}_{n}\right.$. Let $f^{n} \in \mathbf{P L C}([0, R] ;[0, \bar{f}])$ coincide with $f$ on $\mathcal{M}_{n}$. Clearly, $f^{n}$ satisfies $(\mathbf{F})$, because so does $f$. 
Similarly, introduce $\mathbf{P C}\left(\mathbb{R}^{+} ; \mathcal{M}_{n}\right)$, respectively $\mathbf{P C}\left(\mathbb{R}^{+} ; f\left(\mathcal{M}_{n}\right)\right)$, as the sets of piecewise constant functions defined on $\mathbb{R}^{+}$with values in $\mathcal{M}_{n}$, respectively in $f\left(\mathcal{M}_{n}\right)=2^{-n} \mathbb{N} \cup\{\bar{f}\}$. Let $q_{o}^{n}, q_{c}^{n} \in \mathbf{P C}\left(\mathbb{R}^{+} ; f\left(\mathcal{M}_{n}\right)\right)$ coincide with $q_{o}, q_{c}$, on $f\left(\mathcal{M}_{n}\right)$, in the sense that $q_{o}(t)=q_{o}^{n}(t)$ whenever $q_{o}(t) \in f\left(\mathcal{M}_{n}\right)$, respectively $q_{c}(t)=q_{c}^{n}(t)$ whenever $q_{c}(t) \in f\left(\mathcal{M}_{n}\right)$. Note that $q_{o}^{n}, q_{c}^{n}$ satisfy $(\mathbf{Q})$, because so do $q_{o}, q_{c}$. In the same way, let $\rho_{o}^{n}$ be in $\mathbf{P C}\left(\mathbb{R}^{+} ; \mathcal{M}_{n}\right)$ and such that $\rho_{o}^{n}(x)=\rho_{o}(x)$ whenever $\rho_{o}(x) \in \mathcal{M}_{n}$. Clearly, $\rho_{o}^{n}$ satisfies (R0), because so does $\rho_{o}$. The functions $q_{o}^{n}, q_{c}^{n}$ and $\rho_{o}^{n}$ can be represented as

$$
\begin{array}{llll}
\rho_{o}^{n}=\sum_{\alpha \geq 1} \rho_{o \alpha}^{n} \chi_{] x_{o \alpha-1}^{n}, x_{o \alpha}^{n}\right]} & \text { with } & \rho_{\alpha}^{n} \in \mathcal{M}_{n} \\
q_{o}^{n}=\sum_{\beta \geq 1} q_{o \beta}^{n} \chi_{] t_{o \beta-1}^{n}, t_{o \beta}^{n}\right]} & \text { with } & q_{o \beta}^{n} \in f\left(\mathcal{M}_{n}\right) \\
q_{c}^{n}=\sum_{\beta \geq 1} q_{c \beta}^{n} \chi_{] t_{c \beta-1}^{n}, t_{c \beta}^{n}\right]} & \text { with } & q_{c \beta}^{n} \in f\left(\mathcal{M}_{n}\right)
\end{array}
$$

and we agree that $x_{o 0}^{n}=t_{o 0}^{n}=t_{c 0}^{n}=0$. All the approximations above are meant in the strong $\mathbf{L}^{1}$-topology, that is

$$
\lim _{n \rightarrow+\infty}\left(\left\|\rho_{o}^{n}-\rho_{o}\right\|_{\mathbf{L}^{1}(\mathbb{R} ; \mathbb{R})}+\left\|q_{o}^{n}-q_{o}\right\|_{\mathbf{L}^{1}\left(\mathbb{R}^{+} ; \mathbb{R}\right)}+\left\|q_{c}^{n}-q_{c}\right\|_{\mathbf{L}^{1}\left(\mathbb{R}^{+} ; \mathbb{R}\right)}\right)=0 .
$$

We now follow the nowadays classical wave front tracking technique which dates back to [16], which we adopt in the form suited to the scalar case, see [8], Chapter 6, or also [11]. The present construction comprises that in [9]. In particular, an approximate solution $\rho^{n}$ to $(1.2)$ is computed as the exact weak entropy solution to

$$
\left\{\begin{array}{lc}
\partial_{t} \rho^{n}+\partial_{x} f^{n}\left(\rho^{n}\right)=0 & (t, x) \in \mathbb{R}^{+} \times \mathbb{R}^{+} \\
\rho^{n}(0, x)=\rho_{o}^{n}(x) & x \in \mathbb{R}^{+} \\
f^{n}\left(\rho^{n}(t, 0)\right)=q_{o}^{n}(t) & t \in \mathbb{R}^{+} \\
f^{n}\left(\rho^{n}\left(t, x_{c}\right)\right) \leq q_{c}^{n}(t) & t \in \mathbb{R}^{+} .
\end{array}\right.
$$

In other words, $\rho^{n}$ is obtained gluing the solutions to the Riemann problems at the points $\left(0, x_{o \alpha}^{n}\right)$ of jump of $\rho_{o}^{n}$, at the points $\left(t_{o \beta}^{n}, 0\right)$ where the boundary data changes, at the points $\left(t_{c \beta}^{n}, x_{c}\right)$ where the constraint changes and at all interaction points, which are defined here exactly as in [11], Section 4.2. For the definition of the solution to standard Riemann problems with a piecewise linear and continuous flow, we refer to [8], Section 6.1, Cases 1 and 2. The constrained Riemann problem is solved in detail in [11], Section 2. For the solution to the Riemann problem at the boundary, we refer to (4.1) above.

Iteratively solving Riemann problems at the boundary, at interactions and at the constraint, we define a $\rho^{n}$ which is a weak entropy solution to (4.3) and an approximate solution to (1.2).

We prove that $\rho^{n}(t) \in \mathcal{D}_{n}$, where $\mathcal{D}_{n}=\left\{\rho \in \mathbf{P C}\left(\mathbb{R}^{+} ; \mathcal{M}_{n}\right): \Psi(\rho) \in \mathbf{B V}\left(\mathbb{R}^{+} ; \mathbb{R}\right)\right\}$. To this aim, as it is usual in the context of initial-boundary value problems for conservation laws, see for instance [2], introduce the set $\overline{\mathcal{D}}_{n}=$ $\mathcal{D}_{n} \times\left(\mathbf{P C}\left(\mathbb{R}^{+} ; f\left(\mathcal{M}_{n}\right)\right)\right)^{2}$ and a semigroup $\bar{S}^{n}: \mathbb{R}^{+} \times \overline{\mathcal{D}}_{n} \rightarrow \overline{\mathcal{D}}_{n}$ setting $\bar{S}_{t}\left(\rho_{o}^{n}, q_{o}^{n}, q_{c}^{n}\right)=\left(\rho^{n}(t), \mathcal{T}_{t} q_{o}^{n}, \mathcal{T}_{t} q_{c}^{n}\right)$, where $\mathcal{T}_{t}$ is the usual translation operator: $\left(\mathcal{T}_{t} q\right)(\tau)=q(t+\tau)$.

On any $\left(\rho^{n}, q_{o}^{n}, q_{c}^{n}\right) \in \overline{\mathcal{D}}_{n}$, written as in (4.2), define the Glimm type functional

$$
\Upsilon=\sum_{\alpha}\left|\Psi\left(\rho_{\alpha+1}^{n}\right)-\Psi\left(\rho_{\alpha}^{n}\right)\right|+2 \sum_{t_{o \beta}^{n} \geq 0}\left|q_{o \beta+1}^{n}-q_{o \beta}^{n}\right|+5 \sum_{t_{c \beta}^{n} \geq 0}\left|q_{c \beta+1}^{n}-q_{c \beta}^{n}\right|+\gamma_{o}+\gamma_{c}
$$



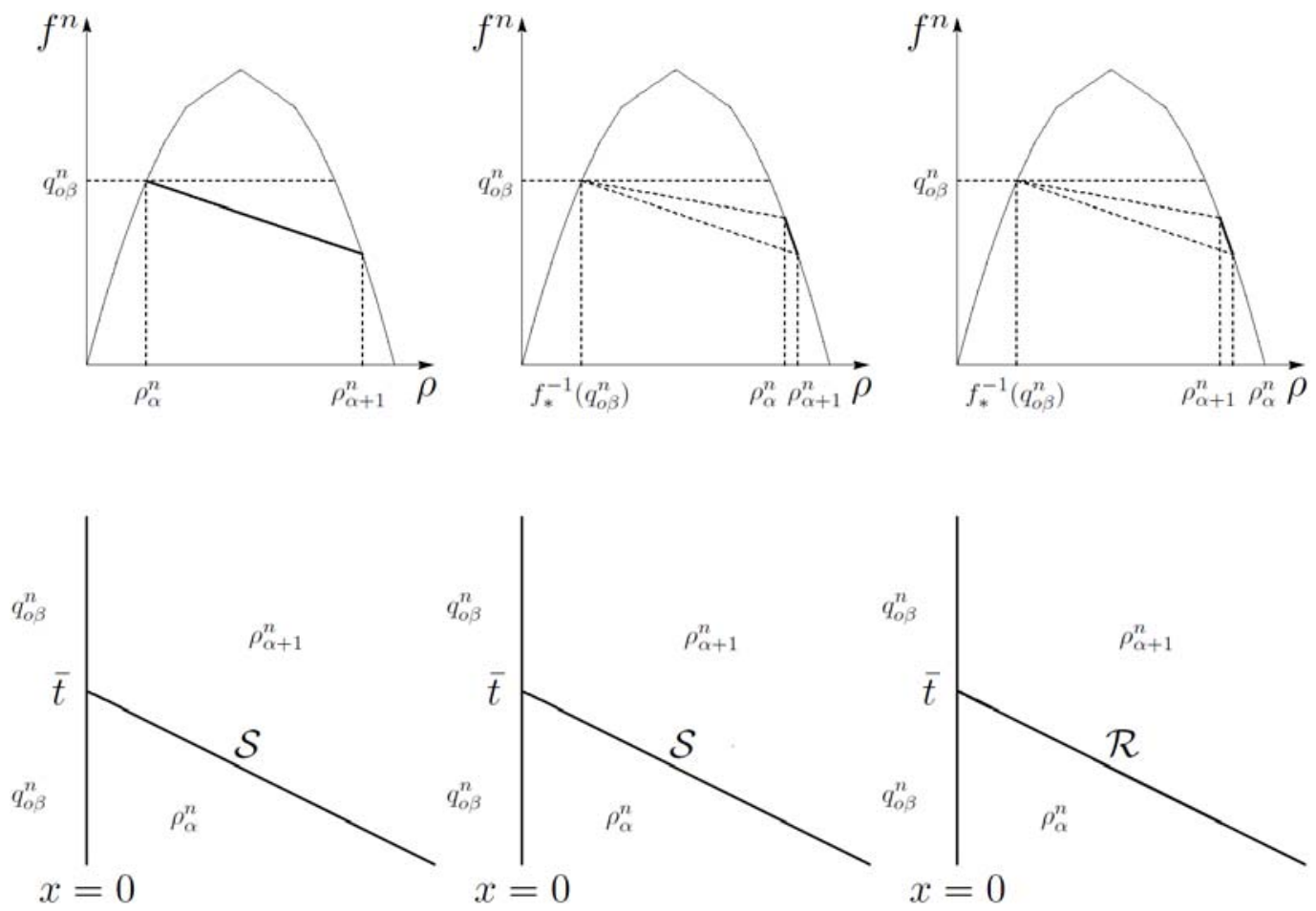

FiguRE 7. Three possible interactions at the boundary when a wave hits it and corresponding to the cases (H1), (H2.1) and (H2.2.1).

where $\Psi$ is as in (2.2) and $\gamma_{o}, \gamma_{c}$ are defined by

$$
\begin{aligned}
& \gamma_{o}= \begin{cases}0 & \text { if } \rho^{n}(0+)=f_{*}^{-1}\left(q_{o}^{n}(0)\right) \\
2\left(f(\bar{\rho})-q_{o}^{n}(0)\right) & \text { otherwise }\end{cases} \\
& \gamma_{c}= \begin{cases}0 & \text { if } \rho^{n}\left(x_{c}-\right)>\bar{\rho}>\rho^{n}\left(x_{c}+\right) \text { and } \\
4\left(f(\bar{\rho})-q_{c}^{n}(0)\right) & \text { otherwise. }\end{cases}
\end{aligned}
$$

Long but elementary computations show that, at any interaction, the functional $\Upsilon$ either decreases by at least $2^{-n}$, or remains constant while the total number of waves in the approximate solution does not increase (this may happen in interactions away from the boundary and the constraint). In particular, a detailed study of the interactions away from the boundary can be found in [11], Section 4 . Therefore, the only further cases to be analyzed are made of waves hitting the boundary or generated by variations in the boundary conditions:

$(\mathbf{H})$ : Assume that a wave between $\rho_{\alpha}^{n}$ and $\rho_{\alpha+1}^{n}$ hits the boundary at time $\left.\left.\bar{t} \in\right] t_{o \beta-1}^{n}, t_{o \beta}^{n}\right]$.

(H1): If $\rho_{\alpha}^{n}=f_{*}^{-1}\left(q_{o \beta}^{n}\right)$, then must be $\rho_{\alpha+1}^{n}>\bar{\rho}$ and $f\left(\rho_{\alpha+1}^{n}\right)<q_{o \beta}^{n}$. In this case the wave is a shock that hits the boundary and disappears, see Figure 7, left. Furthermore

$$
\begin{aligned}
\Upsilon(\bar{t}+)-\Upsilon(\bar{t}-) & =-\left|\psi\left(\rho_{\alpha+1}^{n}\right)-\psi\left(\rho_{\alpha}^{n}\right)\right|+2\left(f(\bar{\rho})-q_{o \beta}^{n}\right) \\
& =-\left(q_{o \beta}^{n}-f\left(\rho_{\alpha+1}^{n}\right)\right) \leq-2^{-n} .
\end{aligned}
$$


(H2): If $\rho_{\alpha}^{n} \neq f_{*}^{-1}\left(q_{o \beta}^{n}\right)$, then must be $\rho_{\alpha}^{n}>\bar{\rho}$ and $f\left(\rho_{\alpha}^{n}\right) \leq q_{o \beta}^{n}$.

(H2.1): If $\rho_{\alpha+1}^{n}>\rho_{\alpha}^{n}$, then the wave is a shock that disappears after hitting the boundary, see Figure 7 , center. In this case

$$
\Upsilon(\bar{t}+)-\Upsilon(\bar{t}-)=-\left|\psi\left(\rho_{\alpha+1}^{n}\right)-\psi\left(\rho_{\alpha}^{n}\right)\right|=-\left(f\left(\rho_{\alpha}^{n}\right)-f\left(\rho_{\alpha+1}^{n}\right)\right) \leq-2^{-n} .
$$

(H2.2): If $\rho_{\alpha+1}^{n}<\rho_{\alpha}^{n}$, then the wave is a rarefaction.

(H2.2.1): If $f\left(\rho_{\alpha+1}^{n}\right) \leq q_{o \beta}^{n}$ then the hitting wave disappears, see Figure 7, right, and

$$
\Upsilon(\bar{t}+)-\Upsilon(\bar{t}-)=-\left|\psi\left(\rho_{\alpha+1}^{n}\right)-\psi\left(\rho_{\alpha}^{n}\right)\right|=-\left(f\left(\rho_{\alpha+1}^{n}\right)-f\left(\rho_{\alpha}^{n}\right)\right) \leq-2^{-n}
$$

(H2.2.2): If $f\left(\rho_{\alpha+1}^{n}\right)>q_{o \beta}^{n}$ then a shock wave arises from the boundary, see Figure 8, left, and

$$
\begin{aligned}
\Upsilon(\bar{t}+)-\Upsilon(\bar{t}-) & =\left|\psi\left(f_{*}^{-1}\left(q_{o \beta}^{n}\right)\right)-\psi\left(\rho_{\alpha+1}^{n}\right)\right|-\left|\psi\left(\rho_{\alpha+1}^{n}\right)-\psi\left(\rho_{\alpha}^{n}\right)\right|-2\left(f(\bar{\rho})-q_{o \beta}^{n}\right) \\
& =-2\left(f\left(\rho_{\alpha+1}^{n}\right)-q_{o \beta}^{n}\right) \leq-2^{-n} .
\end{aligned}
$$

(B): At time $\bar{t}=t_{o \beta-1}^{n}$ the boundary condition changes. Let $\rho_{\alpha}^{n}=\rho\left(t_{o \beta-1}^{n}-, 0+\right)$.

(B1): Assume that $q_{o \beta-1}^{n}<q_{o \beta}^{n}$.

(B1.1): If $\rho_{\alpha}^{n}=f_{*}^{-1}\left(q_{o \beta-1}^{n}\right)$, then a decreasing rarefaction between $\rho_{\alpha+m}^{n}=f_{*}^{-1}\left(q_{o \beta}^{n}\right)$ and $\rho_{\alpha}^{n}$ starts from the boundary at time $\bar{t}$ and takes all the values $\rho_{\alpha+i}^{n}, i=0, \ldots, m$, see Figure 8 , center. In this case

$$
\begin{aligned}
\Upsilon(\bar{t}+)-\Upsilon(\bar{t}-) & =\sum_{i=0}^{m-1}\left|\psi\left(\rho_{\alpha+i}^{n}\right)-\psi\left(\rho_{\alpha+i+1}^{n}\right)\right|-2\left|q_{o \beta}^{n}-q_{o \beta-1}^{n}\right| \\
& =\sum_{i=0}^{m-1}\left(f\left(\rho_{\alpha+i+1}^{n}\right)-f\left(\rho_{\alpha+i}^{n}\right)\right)-2\left(q_{o \beta}^{n}-q_{o \beta-1}^{n}\right) \\
& =-\left(q_{o \beta}^{n}-q_{o \beta-1}^{n}\right) \leq-2^{-n} .
\end{aligned}
$$

(B1.2): If $\rho_{\alpha}^{n} \neq f_{*}^{-1}\left(q_{o \beta-1}^{n}\right)$, then must be $\rho_{\alpha}^{n}>\bar{\rho}$ and $f\left(\rho_{\alpha}^{n}\right) \leq q_{o \beta-1}^{n}$, see Figure 8 , right. In this case no wave appears and $\rho^{n}(\bar{t}, 0+)=\rho_{\alpha}^{n}$. Furthermore

$$
\begin{aligned}
\Upsilon(\bar{t}+)-\Upsilon(\bar{t}-) & =-2\left|q_{o \beta}^{n}-q_{o \beta-1}^{n}\right|+2\left(f(\bar{\rho})-q_{o \beta}^{n}\right)-2\left(f(\bar{\rho})-q_{o \beta-1}^{n}\right) \\
& =-4\left(q_{o \beta}^{n}-q_{o \beta-1}^{n}\right) \leq-2^{-n} .
\end{aligned}
$$

(B2): Assume that $q_{o \beta-1}^{n}>q_{o \beta}^{n}$.

(B2.1): If $\rho_{\alpha}^{n}=f_{*}^{-1}\left(q_{o \beta-1}^{n}\right)$, then an increasing shock between $f_{*}^{-1}\left(q_{o \beta}^{n}\right)$ and $\rho_{\alpha}^{n}$ starts from the boundary at time $\bar{t}$, see Figure 9 , left. The functional $\Upsilon$ changes as follows

$$
\begin{aligned}
\Upsilon(\bar{t}+)-\Upsilon(\bar{t}-) & =\left|\psi\left(f_{*}^{-1}\left(q_{o \beta}^{n}\right)\right)-\psi\left(\rho_{\alpha}^{n}\right)\right|-2\left|q_{o \beta}^{n}-q_{o \beta-1}^{n}\right| \\
& =-\left(q_{o \beta-1}^{n}-q_{o \beta}^{n}\right) \leq-2^{-n} .
\end{aligned}
$$

(B2.2): If $\rho_{\alpha}^{n} \neq f_{*}^{-1}\left(q_{o \beta-1}^{n}\right)$, then must be $\rho_{\alpha}^{n}>\bar{\rho}$ and $f\left(\rho_{\alpha}^{n}\right) \leq q_{o \beta-1}^{n}$. 

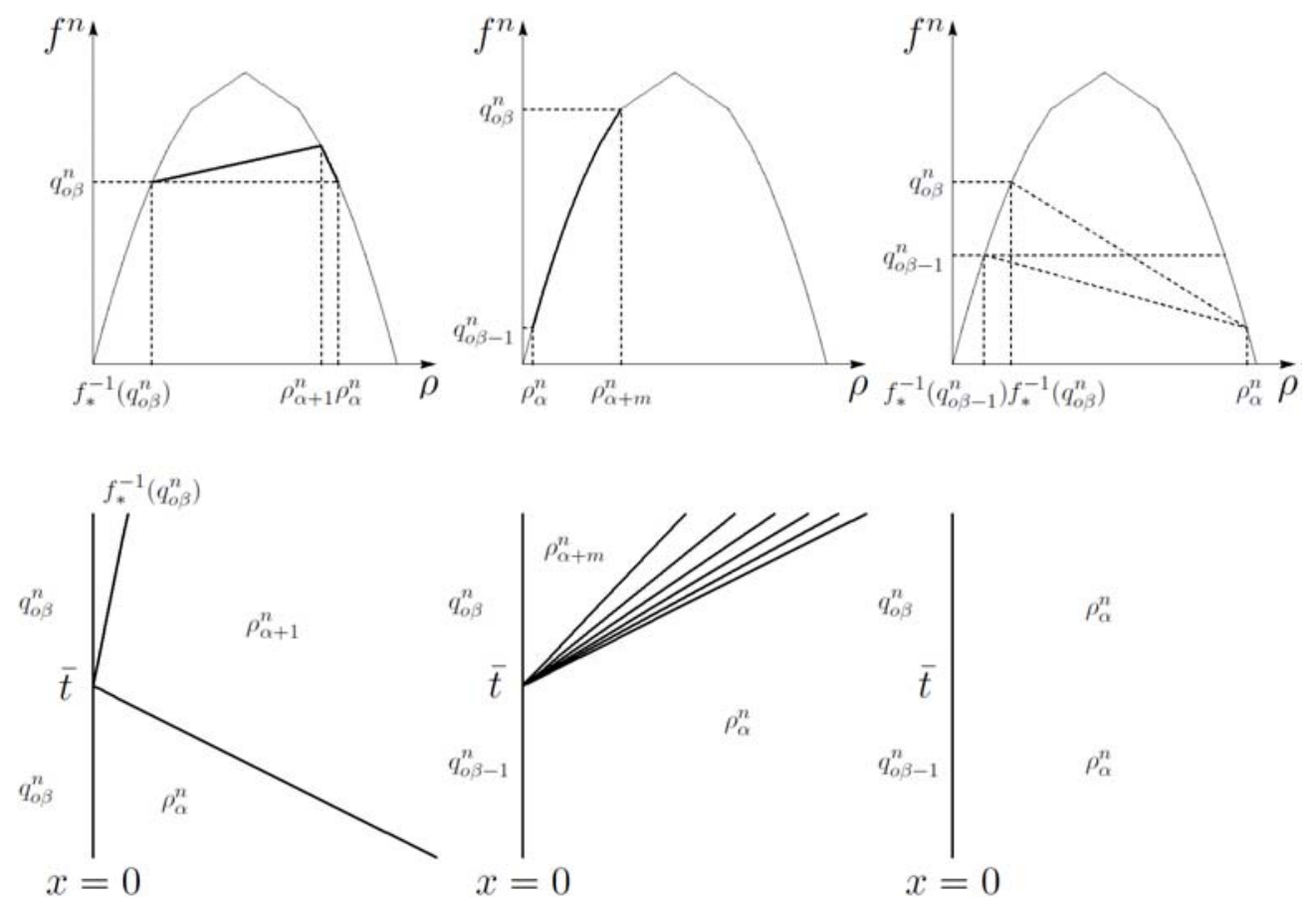

Figure 8. Left: The interaction considered in (H2.2.2). Center and right: The two possible interactions at the boundary when the boundary data increases and corresponding to the cases (B1.1) and (B1.2).

(B2.2.1): If $f\left(\rho_{\alpha}^{n}\right)>q_{o \beta}^{n}$, then an increasing shock between $f_{*}^{-1}\left(q_{o \beta}^{n}\right)$ and $\rho_{\alpha}^{n}$ starts from the boundary at time $\bar{t}$, see Figure 9, center, and

$$
\begin{aligned}
\Upsilon(\bar{t}+)-\Upsilon(\bar{t}-) & =\left|\psi\left(f_{*}^{-1}\left(q_{o \beta}^{n}\right)\right)-\psi\left(\rho_{\alpha}^{n}\right)\right|-2\left|q_{o \beta}^{n}-q_{o \beta-1}^{n}\right|-2\left(f(\bar{\rho})-q_{o \beta-1}^{n}\right) \\
& =-\left(f\left(\rho_{\alpha}^{n}\right)-q_{o \beta}^{n}\right) \leq-2^{-n} .
\end{aligned}
$$

(B2.2.2): If $f\left(\rho_{\alpha}^{n}\right) \leq q_{o \beta}^{n}$, then no wave starts from the boundary, see Figure 9, right, and

$$
\Upsilon(\bar{t}+)-\Upsilon(\bar{t}-)=-2\left|q_{o \beta}^{n}-q_{o \beta-1}^{n}\right|+2\left(f(\bar{\rho})-q_{o \beta}^{n}\right)-2\left(f(\bar{\rho})-q_{o \beta-1}^{n}\right)=0 .
$$

Hence, the map $t \rightarrow \Upsilon\left(\bar{S}_{t}^{n}\left(\rho^{n}, q_{n}^{o}, q_{n}^{c}\right)\right)$ is non increasing. Clearly, $\operatorname{TV}\left(\Psi\left(\rho^{n}\right)\right)+\operatorname{TV}\left(q_{o}^{n}\right)+\operatorname{TV}\left(q_{c}^{n}\right) \leq$ $\Upsilon\left(\rho^{n}, q_{o}^{n}, q_{c}^{n}\right)$, so that Helly's Theorem in the form [8], Theorem 2.4, can be applied proving that $\Psi\left(\rho^{n}\right)$ converges a.e. to a limit $\psi \in \mathbf{C}^{\mathbf{0}, \mathbf{1}}\left(\mathbb{R}^{+} ; \mathbf{B V}\left(\mathbb{R}^{+} ;[-\bar{f}, \bar{f}]\right)\right)$. Since $\Psi$ is invertible, also $\rho^{n}$ converges a.e. to $\rho=\Psi^{-1}(\psi)$ and $\rho \in \mathbf{C}^{\mathbf{0}, \mathbf{1}}\left(\mathbb{R}^{+} ; \mathbf{L}^{\infty}\left(\mathbb{R}^{+} ;[0, R]\right)\right)$. To prove that $\rho$ solves $(1.2)$ in the sense of Definition 2.1 , we first check the validity of $\mathbf{1}$. Due to its local nature, we consider the different lines in $\mathbf{1}$. separately:

(1) If $\operatorname{spt} \varphi$ is separated from $x=0$ and from $x=x_{c}$, we need to deal only with the first two lines, essentially with Kružkov definition of solution [34], Definition 1. They are non negative for any $n$ because, by construction, $\rho^{n}$ is a weak entropy solution to (4.3).

(2) If $\operatorname{spt} \varphi$ intersects the line $x=0$, but not the line $x=x_{c}$, then we deal only with the boundary, in particular with the third line in $\mathbf{1}$. Then, $\rho^{n}$ exactly satisfies the boundary condition, at least for all 

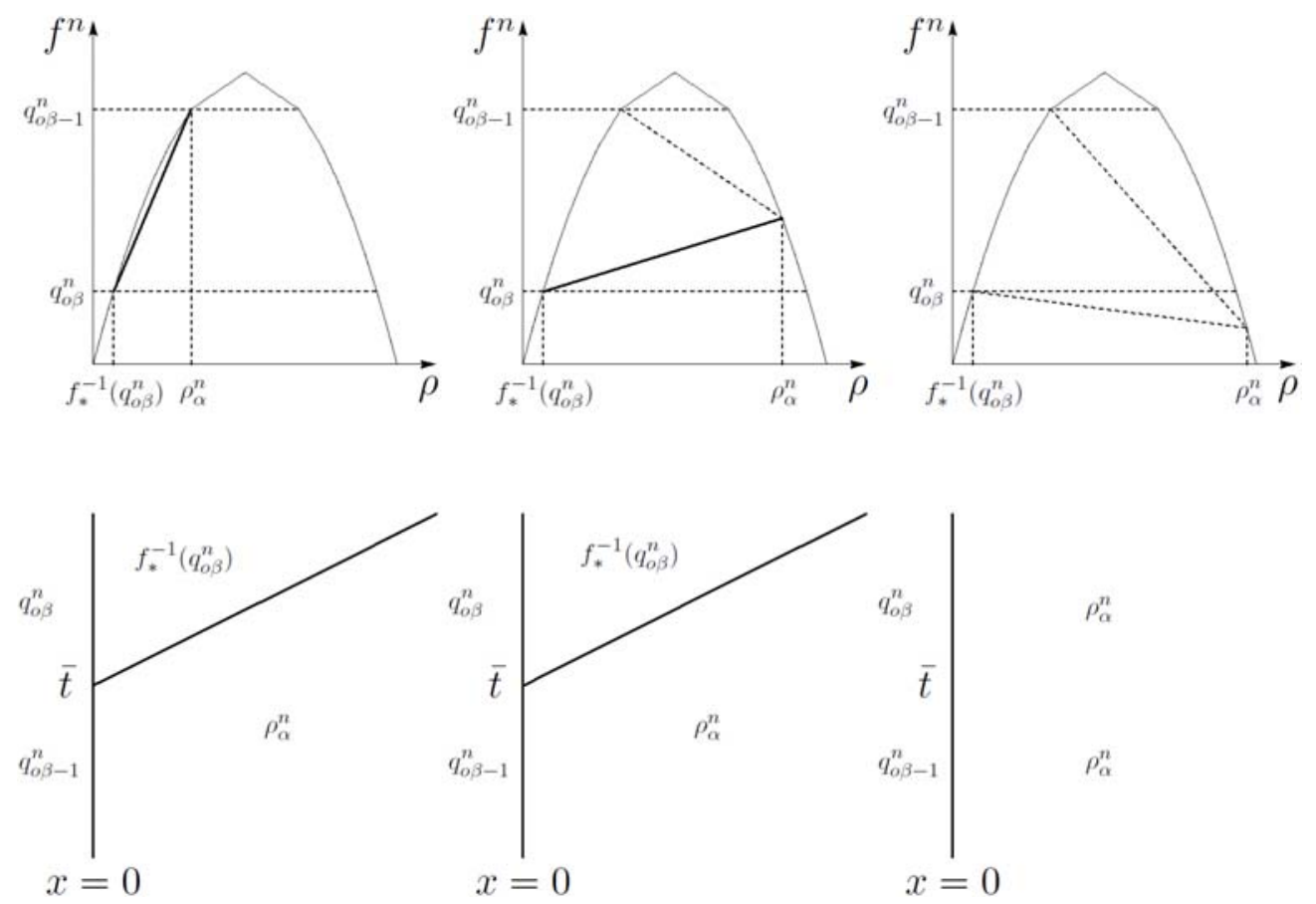

Figure 9. The three possible interactions at the boundary when the boundary data decreases and corresponding to the cases (B2.1), (B2.2.1) and (B2.2.2).

but countably many times. Hence, as in [1], Section 7, [20], Section 3.4, and [44], Theorem 1.4, also at the limit $\rho$ satisfies the boundary condition.

(3) If $\operatorname{spt} \varphi$ intersects the line $x=x_{c}$, but not the line $x=0$, then we are left to verify that $\rho$ satisfies the constraint. This is done exactly as in $[4,11]$.

To verify 2., note first that, by construction, $f\left(\rho^{n}\left(t, x_{c}-\right)\right)=f\left(\rho^{n}\left(t, x_{c}+\right)\right) \leq q_{c}^{n}(t)$ holds for all $n \in \mathbb{N}$ and all but countably many $t \in \mathbb{R}^{+}$. Then, the existence of the traces at the limit follows from [4], Theorem 2.2.

The uniqueness of the solution, as well as the Lipschitz estimate (2.4), is proved by means of the doubling of variables technique, introduced in [34], used in the framework of constrained scalar conservation laws in [4,11] and, in the case with boundary, in [6].

Proof of Corollary 2.3. Fix $t>0$ and $x>x_{c}$. Set

$$
\Phi(u, v)=\operatorname{sgn}(u-v)(f(u)-f(v)) .
$$

Starting from the entropy condition 1. in Definition 2.1, the doubling of variables method applied to the domain $\mathbb{R} \times \mathbb{R}^{+} \backslash\left\{x=x_{c}\right\}$, yields the inequality

$$
\int_{0}^{+\infty} \int_{0}^{+\infty}\left(\left|\rho-\rho^{\prime}\right| \partial_{t} \varphi+\Phi\left(\rho, \rho^{\prime}\right) \partial_{x} \varphi\right) \mathrm{d} y \mathrm{~d} s \geq 0
$$

for all $\varphi \in \mathbf{C}_{\mathbf{c}}^{\mathbf{1}}\left(\left(\mathbb{R}^{+}\right)^{2} \backslash\left(\mathbb{R}^{+} \times\left\{x=x_{c}\right\}\right) ; \mathbb{R}^{+}\right)$. Replacing $\varphi$ by a sequence of approximations $\varphi_{\varepsilon}$ of the characteristic function of the set $] 0, t\left[\times(] 0, x\left[\backslash\left\{x_{c}\right\}\right)\right.$, and passing to the limit as $\varepsilon \rightarrow 0$, we get

$$
\int_{0}^{t} \Phi\left(\rho, \rho^{\prime}\right)(s, x-) \mathrm{d} s+\int_{0}^{x}\left|\rho-\rho^{\prime}\right|(t-, y) \mathrm{d} y \leq \int_{0}^{t} \Phi\left(\rho, \rho^{\prime}\right)(s, 0+) \mathrm{d} s+I I_{x_{c}},
$$


where

$$
I I_{x_{c}}=\int_{0}^{t}\left(\Phi\left(\rho, \rho^{\prime}\right)\left(s, x_{c}+\right)-\Phi\left(\rho, \rho^{\prime}\right)\left(s, x_{c}-\right)\right) \mathrm{d} s \leq 2 \int_{0}^{t}\left|q_{c}-q_{c}^{\prime}\right|(s) \mathrm{d} s .
$$

We now observe that, since the flows are exiting through $x=\bar{x}$, i.e.

$$
\frac{\mathrm{d} f}{\mathrm{~d} \rho}(\rho(t, \bar{x}-)) \geq 0 \quad \text { and } \quad \frac{\mathrm{d} f}{\mathrm{~d} \rho}\left(\rho^{\prime}(t, \bar{x}-)\right) \geq 0
$$

we can deduce the identity

$$
\int_{0}^{t} \Phi\left(\rho, \rho^{\prime}\right)(s, \bar{x}-) \mathrm{d} s=\int_{0}^{t}\left|f(\rho)-f\left(\rho^{\prime}\right)\right|(s, \bar{x}-) \mathrm{d} s .
$$

On the other side, at $x=0$, we have

$$
\int_{0}^{t} \Phi\left(\rho, \rho^{\prime}\right)(s, 0+) \mathrm{d} s \leq \int_{0}^{t}\left|q_{o}-q_{o}^{\prime}\right|(s) \mathrm{d} s .
$$

Combining (4.5), (4.6), (4.7) and (4.8), we get (2.6).

Proof of Corollary 2.4. The proof consists of the following applications of Theorem 2.2.

First, solve the two independent problems

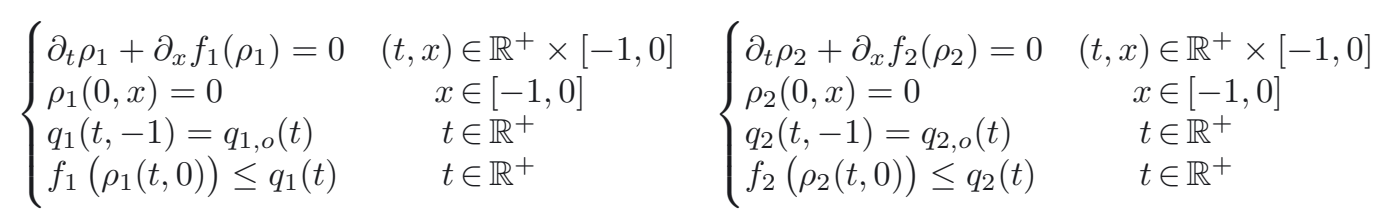

applying Theorem 2.2. Then, add the traces of the two flows from the incoming roads at $x=0$, setting $q_{3, o}(t)=f_{1}\left(\rho_{1}(t, 0-)\right)+f_{2}\left(\rho_{2}(t, 0-)\right)$. Finally solve the problem

$$
\left\{\begin{array}{lrl}
\partial_{t} \rho_{3}+\partial_{x} f_{3}\left(\rho_{3}\right)=0 & (t, x) & \in \mathbb{R}^{+} \times \mathbb{R}^{+} \\
\rho_{3}(0, x)=0 & x \in \mathbb{R}^{+} \\
q_{3}(t, 0)=q_{3, o}(t) & t \in \mathbb{R}^{+}
\end{array}\right.
$$

again by means of Theorem 2.2 .

Proof of Proposition 2.5. Let us consider a sequence of functions $\rho_{\nu} \mathbf{L}^{\mathbf{1}}$-converging to some $\tilde{\rho}$ on $\left[0, x_{c}\right]$ :

$$
\lim _{\nu \rightarrow \infty}\left\|\rho_{\nu}-\tilde{\rho}\right\|_{\mathbf{L}^{1}\left(\left[0, x_{c}\right] ; \mathbb{R}\right)}=0
$$

Fix $\varepsilon>0$. If $A_{c}\left(\rho_{\nu}\right)=\emptyset$ for all $\nu \geq N$, for some $N>0$, then

$$
0=\mathcal{L}\left(\rho_{\nu}\right) \leq \mathcal{L}(\tilde{\rho})+\varepsilon \quad \forall \nu \geq N
$$

Otherwise, there exists a subsequence (again labeled $\left.\rho_{\nu}\right)$ such that $A_{c}\left(\rho_{\nu}\right) \neq \emptyset$ for all $\nu \in \mathbb{N}$. Let $x_{\nu}=\inf A_{c}\left(\rho_{\nu}\right)$ and $\breve{x}=\liminf _{\nu \rightarrow \infty} x_{\nu}$. Therefore, for any $\varepsilon>0$, there exists $N>0$ s.t.

$$
\mathcal{L}\left(\rho_{\nu}\right) \leq x_{c}-\breve{x}+\varepsilon \quad \forall \nu \geq N
$$


and there exists a subsequence (again labeled $x_{\nu}$ ) such that $x_{\nu} \rightarrow \breve{x}+$. Moreover, since $\rho_{\nu}$ converge pointwise a.e. to $\tilde{\rho}$ on $\left[0, x_{c}\right]$ and $\rho_{\nu}(\xi)=\Psi^{-1}\left(\bar{f}-Q_{c}\right)$ for a.e. $\xi \in\left[x_{\nu}, x_{c}\right]$, we get that $\tilde{\rho}(\xi)=\Psi^{-1}\left(\bar{f}-Q_{c}\right)$ for a.e. $\xi \in\left[\breve{x}, x_{c}\right]$. In fact, fix $\varepsilon>0$ : for any $\left.\left.\xi \in\right] \breve{x}, x_{c}\right]$ such that $\lim _{\nu \rightarrow \infty} \rho_{\nu}(\xi)=\tilde{\rho}(\xi)$, there exists $N>0$ sufficiently large such $x_{m}<\xi$ and $\left|\rho_{m}(\xi)-\tilde{\rho}(\xi)\right| \leq \varepsilon$ for all $m \geq N$. Since $\rho_{m}(\xi)=\Psi^{-1}\left(\bar{f}-Q_{c}\right)$ a.e., we have $\left|\tilde{\rho}(\xi)-\Psi^{-1}\left(\bar{f}-Q_{c}\right)\right| \leq \varepsilon$. This implies

$$
\left.\left.\tilde{\rho}(\xi)=\Psi^{-1}\left(\bar{f}-Q_{c}\right) \quad \text { a.e. } \xi \in\right] \breve{x}, x_{c}\right] .
$$

Hence

$$
\mathcal{L}(\tilde{\rho}) \geq x_{c}-\breve{x} \geq \mathcal{L}\left(\rho_{\nu}\right)-\varepsilon \quad \forall \nu \geq N
$$

which concludes the proof.

Proof of Proposition 2.6. Straightforward computations give:

$$
\begin{aligned}
T_{a}-T_{a}^{\prime}= & \frac{1}{Q_{\text {in }}} \int_{0}^{+\infty} t f(\rho(t, \bar{x})) \mathrm{d} t-\frac{1}{Q_{\text {in }}} \int_{0}^{+\infty} t f\left(\rho^{\prime}(t, \bar{x})\right) \mathrm{d} t \\
& +\frac{1}{Q_{\text {in }}} \int_{0}^{+\infty} t f\left(\rho^{\prime}(t, \bar{x})\right) \mathrm{d} t-\frac{1}{Q_{\text {in }}^{\prime}} \int_{0}^{+\infty} t f\left(\rho^{\prime}(t, \bar{x})\right) \mathrm{d} t \\
= & \frac{1}{Q_{\text {in }}}\left(\int_{0}^{+\infty} t f(\rho(t, \bar{x})) \mathrm{d} t-\int_{0}^{+\infty} t f\left(\rho^{\prime}(t, \bar{x})\right) \mathrm{d} t\right) \\
& +\left(\frac{1}{Q_{\text {in }}}-\frac{1}{Q_{\text {in }}^{\prime}}\right) \int_{0}^{+\infty} t f\left(\rho^{\prime}(t, \bar{x})\right) \mathrm{d} t \\
T_{t}-T_{t}^{\prime}= & T_{a}-T_{a}^{\prime}-\frac{1}{Q_{\text {in }}} \int_{0}^{+\infty} t q_{o}(t) \mathrm{d} t+\frac{1}{Q_{\text {in }}^{\prime}} \int_{0}^{+\infty} t q_{o}^{\prime}(t) \mathrm{d} t \\
= & T_{a}-T_{a}^{\prime}-\frac{1}{Q_{\text {in }}} \int_{0}^{+\infty} t q_{o}(t) \mathrm{d} t+\frac{1}{Q_{\text {in }}} \int_{0}^{+\infty} t q_{o}^{\prime}(t) \mathrm{d} t \\
& -\frac{1}{Q_{\text {in }}} \int_{0}^{+\infty} t q_{o}^{\prime}(t) \mathrm{d} t+\frac{1}{Q_{\text {in }}^{\prime}} \int_{0}^{+\infty} t q_{o}^{\prime}(t) \mathrm{d} t \\
= & T_{a}-T_{a}^{\prime}+\frac{1}{Q_{\text {in }}} \int_{0}^{+\infty} t\left(q_{o}^{\prime}(t)-q_{o}(t)\right) \mathrm{d} t+\left(\frac{1}{Q_{\text {in }}^{\prime}}-\frac{1}{Q_{\text {in }}}\right) \int_{0}^{+\infty} t q_{o}^{\prime}(t) \mathrm{d} t .
\end{aligned}
$$

The final estimates now easily follow from Corollary 2.3 .

Acknowledgements. The first and second author were supported by the NUSMAIN-NOMAIN 2009 project of the Galileo program 2009 (French-Italian cooperation program). The first and third author acknowledge the hospitality of INRIA Sophia Antipolis - Méditerranée.

\section{REFERENCES}

[1] D. Amadori, Initial-boundary value problems for nonlinear systems of conservation laws. NoDEA 4 (1997) 1-42.

[2] D. Amadori and R.M. Colombo, Continuous dependence for $2 \times 2$ conservation laws with boundary. J. Differ. Equ. 138 (1997) 229-266.

[3] F. Ancona and A. Marson, Scalar non-linear conservation laws with integrable boundary data. Nonlinear Anal. 35 (1999) $687-710$. 
[4] B. Andreianov, P. Goatin and N. Seguin, Finite volume schemes for locally constrained conservation laws. Numer. Math. 115 (2010) 609-645.

[5] A. Aw and M. Rascle, Resurrection of "second order" models of traffic flow. SIAM J. Appl. Math. 60 (2000) $916-938$.

[6] C. Bardos, A.Y. le Roux and J.-C. Nédélec, First order quasilinear equations with boundary conditions. Comm. Partial Differential Equations 4 (1979) 1017-1034.

[7] S. Blandin, D. Work, P. Goatin, B. Piccoli and A. Bayen, A general phase transition model for vehicular traffic. SIAM J. Appl. Math. (to appear).

[8] A. Bressan, Hyperbolic systems of conservation laws - The one-dimensional Cauchy problem, Oxford Lecture Series in Mathematics and its Applications 20. Oxford University Press, Oxford (2000).

[9] W. Chen, S.C. Wong, C.W. Shu and P. Zhang, Front tracking algorithm for the Lighthill-Whitham-Richards traffic flow model with a piecewise quadratic, continuous, non-smooth and non-concave fundamental diagram. Int. J. Numer. Anal. Model. 6 (2009) 562-585.

[10] R.M. Colombo, Hyperbolic phase transitions in traffic flow. SIAM J. Appl. Math. 63 (2002) 708-721.

[11] R.M. Colombo and P. Goatin, A well posed conservation law with a variable unilateral constraint. J. Differ. Equ. 234 (2007) 654-675.

[12] R.M. Colombo and A. Groli, Minimising stop and go waves to optimise traffic flow. Appl. Math. Lett. 17 (2004) 697-701.

[13] R.M. Colombo, P. Goatin, G. Maternini and M.D. Rosini, Conservation laws with unilateral constraints in traffic modeling, in Transport Management and Land-Use Effects in Presence of Unusual Demand, L. Mussone and U. Crisalli Eds., Atti del convegno SIDT 2009 (2009).

[14] R.M. Colombo, P. Goatin and B. Piccoli, Road networks with phase transitions. J. Hyperbolic Differ. Equ. 7 (2010) 85-106.

[15] R.M. Colombo, F. Marcellini and M. Rascle, A 2-phase traffic model based on a speed bound. SIAM J. Appl. Math. 70 (2010) 2652-2666.

[16] C.M. Dafermos, Polygonal approximations of solutions of the initial value problem for a conservation law. J. Math. Anal. Appl. 38 (1972) 33-41.

[17] C. Daganzo, The cell transmission model: A dynamic representation of highway traffic consistent with the hydrodynamic theory. Transp. Res. B 28B (1994) 269-287.

[18] F. Dubois and P. LeFloch, Boundary conditions for nonlinear hyperbolic systems of conservation laws. J. Differential Equations 71 (1988) 93-122.

[19] P. Goatin, The Aw-Rascle vehicular traffic flow model with phase transitions. Math. Comput. Model. 44 (2006) $287-303$.

[20] J. Goodman, Initial Boundary Value Problems for Hyperbolic Systems of Conservation Laws. Ph.D. thesis, California University (1982).

[21] H. Greenberg, An analysis of traffic flow. Oper. Res. 7 (1959) 79-85.

[22] B. Greenshields, A study of traffic capacity. Proceedings of the Highway Research Board 14 (1935) 448-477.

[23] B. Haut, G. Bastin and Y. Chitour, A macroscopic traffic model for road networks with a representation of the capacity drop phenomenon at the junctions, in Proceedings 16th IFAC World Congress, Prague, Czech Republic, July (2005) Tu-M01-TP/3.

[24] D. Helbing, S. Lämmer and J.-P. Lebacque, Self-Organized Control of Irregular or Perturbed Network Traffic, in Optimal Control and Dynamic Games, Advances in Computational Management Science 7, Springer (2005) 239-274.

[25] J.C. Herrera and A.M. Bayen, Incorporation of lagrangian measurements in freeway traffic state estimation. Transp. Res. Part B: Methodol. 44 (2010) 460-481.

[26] H. Holden and N.H. Risebro, Front tracking for hyperbolic conservation laws, Applied Mathematical Sciences 152. SpringerVerlag, New York (2002).

[27] W.-L. Jin, Continuous kinematic wave models of merging traffic flow. Transp. Res. Part B: Methodol. 44 (2010) $1084-1103$.

[28] W.L. Jin and H.M. Zhang, The formation and structure of vehicle clusters in the Payne-Whitham traffic flow model. Transp. Res. B 37 (2003) 207-223.

[29] W.L. Jin and H.M. Zhang, On the distribution schemes for determining flows through a merge. Transp. Res. Part B: Methodol. 37 (2003) 521-540.

[30] B.S. Kerner and P. Konhäuser, Cluster effect in initially homogeneous traffic flow. Phys. Rev. E 48 (1993) R2335-R2338.

[31] B.S. Kerner and P. Konhäuser, Structure and parameters of clusters in traffic flow. Phys. Rev. E 50 (1994) $54-83$.

[32] B.S. Kerner and H. Rehborn, Experimental features and characteristics of traffic jams. Phys. Rev. E 53 (1996) R1297-R1300.

[33] A. Klar, Kinetic and Macroscopic Traffic Flow Models. School of Computational Mathematics: Computational aspects in kinetic models, XXth edition (2002).

[34] S.N. Kružhkov, First order quasilinear equations with several independent variables. Mat. Sb. (N.S.) 81 (1970) $228-255$.

[35] L. Leclercq, Bounded acceleration close to fixed and moving bottlenecks. Transp. Res. Part B: Methodol. 41 (2007) $309-319$.

[36] L. Leclercq, Hybrid approaches to the solutions of the Lighthill-Whitham-Richards model. Transp. Res. Part B: Methodol. 41 (2007) 701-709.

[37] H. Lee, H.-W. Lee and D. Kim, Empirical phase diagram of traffic flow on highways with on-ramps, in Traffic and Granular Flow '99, M.S.D.W.D. Helbing and H.J. Herrmann Eds. (2000).

[38] R.J. LeVeque, Finite volume methods for hyperbolic problems. Cambridge Texts in Applied Mathematics, Cambridge University Press, Cambridge (2002). 
[39] J. Li, Q. Chen, H. Wang and D. Ni, Analysis of LWR model with fundamental diagram subject to uncertainties, in TRB 88th Annual Meeting Compendium of Papers, number 09-1189 in TRB (2009) 14.

[40] M.J. Lighthill and G.B. Whitham, On kinematic waves. II. A theory of traffic flow on long crowded roads. Proc. Roy. Soc. London. Ser. A. 229 (1955) 317-345.

[41] H.X. Liu, X. Wu, W. Ma and H. Hu, Real-time queue length estimation for congested signalized intersections. Transp. Res. Part C 17 (2009) 412-427.

[42] S. Mammar, J.-P. Lebacque and H.H. Salem, Riemann problem resolution and Godunov scheme for the Aw-Rascle-Zhang model. Transp. Sci. 43 (2009) 531-545.

[43] G. Newell, A simplified theory of kinematic waves in highway traffic, part II. Transp. Res. B 27 B (1993) $289-303$.

[44] E.Y. Panov, Existence of strong traces for quasi-solutions of multidimensional conservation laws. J. Hyperbolic Differ. Equ. 4 (2007) 729-770.

[45] B. Piccoli and M. Garavello, Traffic flow on networks - Conservation laws models, AIMS Series on Applied Mathematics 1. American Institute of Mathematical Sciences (AIMS), Springfield, MO (2006).

[46] P.I. Richards, Shock waves on the highway. Oper. Res. 4 (1956) 42-51.

[47] D. Serre, Systems of conservation laws 1 \&3 2. Cambridge University Press, Cambridge (1999).

[48] C. Tampere, S. Hoogendoorn and B. van Arem, A behavioural approach to instability, stop \& go waves, wide jams and capacity drop, in Proceedings of 16th International Symposium on Transportation and Traffic Theory (ISTTT), Maryland (2005).

[49] B. Temple, Global solution of the Cauchy problem for a class of $2 \times 2$ nonstrictly hyperbolic conservation laws. Adv. Appl. Math. 3 (1982) 335-375.

[50] E. Tomer, L. Safonov, N. Madar and S. Havlin, Optimization of congested traffic by controlling stop-and-go waves. Phys. Rev. E 65 (2002) 4.

[51] M. Treiber, A. Hennecke and D. Helbing, Congested traffic states in empirical observations and microscopic simulation. Phys. Rev. E 62 (2000) 1805-1824. 